\title{
Single Isomer Iodochlorination of Alkynes and Chlorination of Alkenes Using Tetrabutylammonium Iodide and Dichloroethane
}

\author{
Michael L. Ho, Alison B. Flynn, William W. Ogilvie*
}

Department of Chemistry, University of Ottawa, 10 Marie Curie, Ottawa, Ontario, Canada K1N 6N5

Email:wogilvie@science.uottawa.ca

\section{Table of Contents}

General S1

Synthetic Methods S2

General procedure for the halogenation of alkenes and alkynes using Bu4NI/DCE.. S2

General procedure for the halogenation of alkenes and alkynes using ICl................. S6

Summary of variable $\mathrm{Bu}_{4} \mathrm{NI}$ amount control reactions:............................................. S10

NMR Spectra

General. Reactions were performed under nitrogen in oven-dried glassware equipped with a magnetic stirbar and a rubber septum unless otherwise indicated. Solvents were freshly distilled prior to use as follows: THF over sodium/benzophenone; dichloroethane, dichloromethane and DMF over calcium hydride. Triethylamine and diisopropylethylamine were freshly distilled over calcium hydride. All other were purchased and used without further purification unless otherwise indicated. Reactions were monitored by TLC analysis using glass plates precoated ( $250 \mu \mathrm{m}$ thickness) with silica gel 60 F254. TLC plates were visualized using ultraviolet light and potassium permanganate stain. Flash chromatography was carried out on 230-400 mesh silica gel 60 , or preparatory TLC glass plates precoated with silica gel (Si250F). NMR spectra 
were acquired on $300 \mathrm{MHz}, 400 \mathrm{MHz}$ or $500 \mathrm{MHz}$ spectrometers in the specified solvent. Infrared spectra were acquired on a FTIR spectrometer.

\section{Synthetic Methods}

\section{General procedure for the halogenation of alkenes and alkynes using $\mathrm{Bu}_{4} \mathrm{NI} / \mathrm{DCE}$.}

(E)-Methyl 3-chloro-2-iodoacrylate (9). ${ }^{1}$ A solution of methyl-2-propiolate 4 (250 $\mathrm{mg}, 2.98 \mathrm{mmol}, 1.0$ equiv) and tetrabutylammonium iodide $(3.25 \mathrm{~g}, 8.95 \mathrm{mmol}, 3.0$ equiv) in dichloroethane $(25 \mathrm{~mL})$ was heated at reflux for $18 \mathrm{~h}$. The reaction mixture was cooled, diluted with $\mathrm{Et}_{2} \mathrm{O}$ and washed with $\mathrm{NaHSO}_{3}$ (20\% wt solution), saturated $\mathrm{NaHCO}_{3}$ and brine. The organic phase was then dried over anhydrous $\mathrm{MgSO}_{4}$, filtered and concentrated in vacuo. The pure product was obtained by flash chromatography eluting with hexanes then $5 \%$ EtOAc in hexanes to give the title product as a colorless oil (528 mg, $72 \%$ ). ${ }^{1} \mathrm{H}$ NMR (300 MHz, $\left.\mathrm{CDCl}_{3}\right) \delta 7.00$ (s, 1H), 3.79 (s, 3H); ${ }^{13} \mathrm{C}$ NMR $\left(75 \mathrm{MHz}, \mathrm{CDCl}_{3}\right) \delta 162.6(\mathrm{C}), 129.4(\mathrm{CH}), 84.3(\mathrm{C}), 53.2\left(\mathrm{CH}_{3}\right)$; IR (neat) 1728, $1567 \mathrm{~cm}^{-1}$; MS (EI) $246\left(\mathrm{M}^{+}\right)$; HRMS Calcd for $\mathrm{C}_{4} \mathrm{H}_{4} \mathrm{ClIO}_{2}\left(\mathrm{M}^{+}\right)$245.8945, found 245.8926.

(E)-Ethyl 4-(tert-butyldimethylsilyloxy)-3-chloro-2-iodobut-2-enoate (17). Prepared from ethyl 4-(tert-butyldimethylsilyloxy)but-2-ynoate (100 $\mathrm{mg}, 0.41 \mathrm{mmol})$ using a procedure similar to that described above for compound $\mathbf{9}$ that provided the title compound as a colorless oil (152 mg, $92 \%) .{ }^{1} \mathrm{H}$ NMR (400 MHz, $\left.\mathrm{CDCl}_{3}\right) \delta 4.53(\mathrm{~s}, 2 \mathrm{H})$, 4.31 (q, $J=7.2 \mathrm{~Hz}, 2 \mathrm{H}), 1.34$ (t, $J=7.2 \mathrm{~Hz}, 3 \mathrm{H}), 0.93$ (s, 9H), 0.13 (s, 6H); ${ }^{13} \mathrm{C}$ NMR $\left(100 \mathrm{MHz}, \mathrm{CDCl}_{3}\right) \delta 164.6(\mathrm{C}), 137.1(\mathrm{C}), 80.1(\mathrm{C}), 68.6\left(\mathrm{CH}_{2}\right), 62.6\left(\mathrm{CH}_{2}\right), 25.8\left(\mathrm{CH}_{3}\right)$, 18.3 (C), $13.9\left(\mathrm{CH}_{3}\right)$; IR (neat) 2956, 1733, $1472 \mathrm{~cm}^{-1}$; HRMS Calcd for $\mathrm{C}_{8} \mathrm{H}_{13} \mathrm{ClIO}_{3} \mathrm{Si}$ 
$\left(\mathrm{M}^{+}-t-\mathrm{Bu}\right) 346.9367$, found 346.9352; Calcd for $\mathrm{C}_{11} \mathrm{H}_{19} \mathrm{ClIO}_{3} \mathrm{Si}\left(\mathrm{M}^{+}-\mathrm{CH}_{3}\right)$ 388.9837, found 388.9830 .

N-Methoxy-N-methylnon-2-ynamide (25). To a solution of 1-hexyne (2.6 mL, 17.7 mmol, 1.0 equiv) in hexanes $(150 \mathrm{~mL})$ at $-78{ }^{\circ} \mathrm{C}$ was added a solution of butyllithium (2.26 M in THF, $8.63 \mathrm{~mL}, 19.5 \mathrm{mmol}, 1.1$ equiv). After 1 hour a solution of N-methoxy$\mathrm{N}$-methylcarbamoyl chloride ${ }^{2}$ (2.40 g, $19.4 \mathrm{mmol}, 1.1$ equiv) in THF $(20 \mathrm{~mL})$ was slowly added via canula. The reaction was stirred for 1 hour at $-78{ }^{\circ} \mathrm{C}$ then allowed to warm to room temperature for 1 hour. The reaction was quenched by the dropwise addition of 10 $\% \mathrm{HCl}$ and diluted with ether. The organic layer was washed sequentially with a saturated solution of sodium bicarbonate and brine, then dried over anhydrous $\mathrm{MgSO}_{4}$, filtered and concentrated. The pure product was obtained by chromatography eluting with 5\% EtOAc in hexanes then 20\% EtOAc in hexanes to give the title product as a pale yellow oil (3.02 g, 77\%). ${ }^{1} \mathrm{H}$ NMR (400 MHz, $\left.\mathrm{CDCl}_{3}\right) \delta 3.40$ (s, 3H), $2.94(\mathrm{~s}, 3 \mathrm{H}), 2.03$ (t, $J=6.4 \mathrm{~Hz}, 2 \mathrm{H}), 1.36-1.14(\mathrm{~m}, 8 \mathrm{H}), 0.93(\mathrm{t}, J=6.8 \mathrm{~Hz}, 3 \mathrm{H}) ;{ }^{13} \mathrm{C} \mathrm{NMR}(100 \mathrm{MHz}$, $\left.\mathrm{CDCl}_{3}\right) \delta 73.0\left(\mathrm{CH}_{3}\right), 61.8\left(\mathrm{CH}_{3}\right), 31.1\left(\mathrm{CH}_{2}\right), 30.8\left(\mathrm{CH}_{2}\right), 28.4\left(\mathrm{CH}_{2}\right), 27.6\left(\mathrm{CH}_{2}\right), 22.4$ $\left(\mathrm{CH}_{2}\right), 18.8\left(\mathrm{CH}_{2}\right), 13.6\left(\mathrm{CH}_{3}\right)$; IR (neat) $2237,1644 \mathrm{~cm}^{-1}$; HRMS Calcd for $\mathrm{C}_{11} \mathrm{H}_{19} \mathrm{NO}_{2}$ $\left(\mathrm{M}^{+}\right)$197.1416, found 197.1405.

(E)-3-Chloro-2-iodo-N-methoxy-N-methylnon-2-enamide (26). Prepared from Nmethoxy-N-methylnon-2-ynamide $(3.02 \mathrm{~g}, 15.3 \mathrm{mmol})$ using a procedure similar to that described above for compound 9 that provided the title compound as a colorless oil (5.05 g, $92 \%$ ). ${ }^{1} \mathrm{H}$ NMR (400 MHz, $\left.\mathrm{C}_{6} \mathrm{D}_{6}\right) \delta 3.22$ (s, 3H), 2.82 (s, 3H), 2.46 (br s, $\left.2 \mathrm{H}\right), 1.48$ $1.45(\mathrm{~m}, 2 \mathrm{H}), 1.19-1.14(\mathrm{~m}, 6 \mathrm{H}), 0.84(\mathrm{t}, J=6.8 \mathrm{~Hz}, 3 \mathrm{H}) ;{ }^{13} \mathrm{C} \mathrm{NMR}\left(100 \mathrm{MHz}, \mathrm{C}_{6} \mathrm{D}_{6}\right) \delta$ $166.3(\mathrm{C}), 135.1(\mathrm{C}), 82.7(\mathrm{C}), 60.8\left(\mathrm{CH}_{3}\right), 40.4\left(\mathrm{CH}_{2}\right), 32.4\left(\mathrm{CH}_{3}\right), 31.8\left(\mathrm{CH}_{2}\right), 28.3$ 
$\left(\mathrm{CH}_{2}\right), 27.1\left(\mathrm{CH}_{2}\right), 22.8\left(\mathrm{CH}_{2}\right), 14.1\left(\mathrm{CH}_{3}\right)$; IR (neat) 2954, 2930, 2858, 1654, $1459 \mathrm{~cm}^{-1}$; HRMS Calcd for $\mathrm{C}_{11} \mathrm{H}_{10} \mathrm{ClIO}_{2}\left(\mathrm{M}^{+}\right)$359.0149, found 359.0201.

(E)-5-Chloro-6-iododec-5-ene (28). Prepared from 5-decyne $(0.13 \mathrm{~mL}, 0.76 \mathrm{mmol})$ using a procedure similar to that described above for compound $\mathbf{9}$ that provided the title compound as a colorless oil (210 mg, $92 \%) .{ }^{1} \mathrm{H}$ NMR (400 MHz, acetone-d 6 ) $\delta 2.68(\mathrm{t}, J$ $=7.2 \mathrm{~Hz}, 4 \mathrm{H}), 1.60-1.48(\mathrm{~m}, 4 \mathrm{H}), 1.39-1.32(\mathrm{~m}, 4 \mathrm{H}), 0.94(\mathrm{t}, J=7.2 \mathrm{~Hz}, 3 \mathrm{H}), 0.93(\mathrm{t}$, $J=7.2 \mathrm{~Hz}, 3 \mathrm{H}) ;{ }^{13} \mathrm{C}$ NMR $\left(100 \mathrm{MHz}\right.$, acetone-d $\left.\mathrm{d}_{6}\right) \delta 133.0(\mathrm{C}), 100.6(\mathrm{C}), 44.3\left(\mathrm{CH}_{2}\right)$, 43.2 $\left(\mathrm{CH}_{2}\right), 32.2\left(\mathrm{CH}_{2}\right), 30.9\left(\mathrm{CH}_{2}\right), 23.3\left(\mathrm{CH}_{2}\right), 23.0\left(\mathrm{CH}_{2}\right), 15.2\left(\mathrm{CH}_{3}\right)$, IR (neat) 2957, 2860, 1623, $1464 \mathrm{~cm}^{-1}$; MS (EI) $300\left(\mathrm{M}^{+}\right)$; HRMS Calcd for $\mathrm{C}_{10} \mathrm{H}_{18} \mathrm{ClI}\left(\mathrm{M}^{+}\right)$300.0142, found 300.0132 .

\section{(E)-(2-Chloro-3-iodobut-2-ene-1,4-diyl)bis(oxy)bis(methylene)dibenzene}

(30).

Prepared from 1,4-bis(benzyloxy)but-2-yne ${ }^{3}(50 \mathrm{mg}, 0.19 \mathrm{mmol})$ using a procedure similar to that described above for compound 9 that provided the title compound as a colorless oil (75 mg, $93 \%) .{ }^{1} \mathrm{H}$ NMR (400 MHz, acetone- $\left.\mathrm{d}_{6}\right) \delta 7.42-7.29(\mathrm{~m}, 10 \mathrm{H})$, $4.57(\mathrm{~s}, 2 \mathrm{H}), 4.55(\mathrm{~s}, 2 \mathrm{H}), 4.51(\mathrm{~s}, 2 \mathrm{H}), 4.44(\mathrm{~s}, 2 \mathrm{H}) ;{ }^{13} \mathrm{C}$ NMR $\left(100 \mathrm{MHz}\right.$, acetone- $\left.\mathrm{d}_{6}\right) \delta$ $139.9(\mathrm{C}), 139.8(\mathrm{C}), 132.7(\mathrm{C}), 130.03(\mathrm{CH}), 130.01(\mathrm{CH}), 129.63(\mathrm{CH}), 129.56(\mathrm{CH})$, $129.41(\mathrm{CH}), 129.38(\mathrm{CH}), 102.6(\mathrm{C}), 77.9\left(\mathrm{CH}_{2}\right), 74.8\left(\mathrm{CH}_{2}\right), 73.5\left(\mathrm{CH}_{2}\right), 73.2\left(\mathrm{CH}_{2}\right)$; IR (nujol) 2957, 1640, $1458 \mathrm{~cm}^{-1}$; MS (EI) $428\left(\mathrm{M}^{+}\right)$; HRMS Calcd for $\mathrm{C}_{18} \mathrm{H}_{18} \mathrm{ClIO}_{2}\left(\mathrm{M}^{+}\right)$ 428.0040, found 428.0043 .

(2,3-Dichloropropoxy)benzene (33). ${ }^{4}$ Prepared from allyloxybenzene $(100 \mathrm{mg}, 0.75$ mmol) using a procedure similar to that described above for compound 9 that provided the title compound as a colorless oil (151 mg, 99\%). ${ }^{1} \mathrm{H}$ NMR (400 MHz, $\left.\mathrm{CDCl}_{3}\right) \delta 7.34$ $-7.31(\mathrm{~m}, 2 \mathrm{H}), 7.05-6.94(\mathrm{~m}, 3 \mathrm{H}), 4.41-4.35(\mathrm{~m}, 1 \mathrm{H}), 4.30(\mathrm{dd}, J=6.4,1.2 \mathrm{~Hz}, 2 \mathrm{H})$, 
$4.02-3.89(\mathrm{~m}, 2 \mathrm{H}) ;{ }^{13} \mathrm{C} \mathrm{NMR}\left(100 \mathrm{MHz}, \mathrm{CDCl}_{3}\right) \delta 157.9(\mathrm{C}), 129.6(\mathrm{CH}), 121.6(\mathrm{CH})$, 114.7 (CH), $68.1\left(\mathrm{CH}_{2}\right), 57.3(\mathrm{CH}), 45.0\left(\mathrm{CH}_{2}\right)$; IR (neat) 2959, 2933, 1599, $1496 \mathrm{~cm}^{-1}$; MS (EI) $204\left(\mathrm{M}^{+}\right)$; HRMS Calcd for $\mathrm{C}_{9} \mathrm{H}_{10} \mathrm{Cl}_{2} \mathrm{O}\left(\mathrm{M}^{+}\right)$204.0109, found 204.0105.

4,5-Dichloropentyl benzoate (36). Prepared from pent-4-enyl benzoate ${ }^{5}(100 \mathrm{mg}, 0.53$ mmol) using a procedure similar to that described above for compound 9 that provided the title compound as a colorless oil (118 mg, 99\%). ${ }^{1} \mathrm{H}$ NMR (400 MHz, $\left.\mathrm{CDCl}_{3}\right) \delta 8.05$ (dd, $J=6.4,1.2 \mathrm{~Hz}, 2 \mathrm{H}), 7.55-7.54(\mathrm{~m}, 1 \mathrm{H}), 7.44(\mathrm{dd}, J=8.0,1.6 \mathrm{~Hz}, 2 \mathrm{H}), 4.38-4.35$ (m, 2H), $4.13-4.10(\mathrm{~m}, 1 \mathrm{H}), 3.79$ (dd, $J=11.0,5.0 \mathrm{~Hz}, 1 \mathrm{H}), 3.66(\mathrm{dd}, J=11.4,7.8 \mathrm{~Hz}$, 1H), $2.22-1.84(\mathrm{~m}, 4 \mathrm{H}) ;{ }^{13} \mathrm{C}$ NMR (100 MHz, $\left.\mathrm{CDCl}_{3}\right) \delta 166.4(\mathrm{C}), 132.9(\mathrm{CH}), 130.0$ (C), $129.5(\mathrm{CH}), 128.3(\mathrm{CH}), 63.9\left(\mathrm{CH}_{2}\right), 60.4(\mathrm{CH}), 47.9\left(\mathrm{CH}_{2}\right), 31.7\left(\mathrm{CH}_{2}\right), 25.2\left(\mathrm{CH}_{2}\right)$; IR (neat) 2956, 2849, 1718, $1451 \mathrm{~cm}^{-1}$; MS (EI) $260\left(\mathrm{M}^{+}\right)$; HRMS Calcd for $\mathrm{C}_{12} \mathrm{H}_{14} \mathrm{ClO}_{2}$ $\left(\mathrm{M}^{+}-\mathrm{Cl}\right)$ 225.0682, found 225.0700.

2-(2,3-Dichloropropyl)phenol (39). Prepared from 2-allylphenol ${ }^{6}$ (100 mg, 0.74 mmol) using a procedure similar to that described above for compound 9 that provided the title compound as a colorless oil (135 mg, 89\%). ${ }^{1} \mathrm{H} \mathrm{NMR}\left(400 \mathrm{MHz}, \mathrm{CDCl}_{3}\right) \delta 7.23$ - $7.16(\mathrm{~m}, 2 \mathrm{H}), 6.94(\mathrm{dd}, J=7.4,7.4 \mathrm{~Hz}, 1 \mathrm{H}), 6.79(\mathrm{~d}, J=8.0 \mathrm{~Hz}, 1 \mathrm{H}), 5.42($ br s, $1 \mathrm{H})$, $4.53-4.66(\mathrm{~m}, 1 \mathrm{H}), 3.78(\mathrm{dd}, J=11.7,5.5 \mathrm{~Hz}, 1 \mathrm{H}), 3.76(\mathrm{dd}, J=11.7,5.36 \mathrm{~Hz}, 1 \mathrm{H})$, $3.35(\mathrm{dd}, J=14.0,6.0 \mathrm{~Hz}, 1 \mathrm{H}), 3.09(\mathrm{dd}, J=14.0,7.6 \mathrm{~Hz}, 1 \mathrm{H}) ;{ }^{13} \mathrm{C} \mathrm{NMR}(100 \mathrm{MHz}$, $\left.\mathrm{CDCl}_{3}\right) \delta 153.6(\mathrm{C}), 131.8(\mathrm{CH}), 128.6(\mathrm{CH}), 123.1(\mathrm{C}), 120.9(\mathrm{CH}), 115.5(\mathrm{CH}), 60.3$ (CH), $48.4\left(\mathrm{CH}_{2}\right), 36.5\left(\mathrm{CH}_{2}\right)$; IR (neat) 3540, 2951, 1609, $1502 \mathrm{~cm}^{-1}$; MS (EI) $204\left(\mathrm{M}^{+}\right)$; HRMS Calcd for $\mathrm{C}_{9} \mathrm{H}_{10} \mathrm{Cl}_{2} \mathrm{O}\left(\mathrm{M}^{+}\right)$204.0109, found 204.0121.

Butyl 2,3-dichloropropanoate ${ }^{7}$ (48): Prepared from $n$-butyl acrylate (50 mg, 0.39 mmol) using a procedure similar to that described above for compound 9 that provided 
the title compound as a colorless oil (15.5 mg, 20\%). ${ }^{1} \mathrm{H}$ NMR $\left(400 \mathrm{MHz}, \mathrm{CDCl}_{3}\right) \delta 4.42$ (dd, $J=8.7,5.2 \mathrm{~Hz}, 1 \mathrm{H}), 4.24(\mathrm{t}, J=6.8 \mathrm{~Hz}, 2 \mathrm{H}), 3.96(\mathrm{dd}, J=11.1,8.7 \mathrm{~Hz}, 1 \mathrm{H}), 3.80$ (dd, $J=11.1,5.2 \mathrm{~Hz}, 1 \mathrm{H}), 1.71-1.64(\mathrm{~m}, 2 \mathrm{H}), 1.44-1.39(\mathrm{~m}, 2 \mathrm{H}), 0.95(\mathrm{t}, J=7.6 \mathrm{~Hz}$, $3 \mathrm{H}) ;{ }^{13} \mathrm{C}$ NMR $\left(100 \mathrm{MHz}, \mathrm{CDCl}_{3}\right) \delta 167.1(\mathrm{C}), 66.5\left(\mathrm{CH}_{2}\right), 55.1(\mathrm{CH}), 43.9\left(\mathrm{CH}_{2}\right), 30.4$ $\left(\mathrm{CH}_{2}\right), 18.9\left(\mathrm{CH}_{2}\right), 13.6\left(\mathrm{CH}_{3}\right)$; IR (neat) 2936, $1750 \mathrm{~cm}^{-1}$.

\section{General procedure for the halogenation of alkenes and alkynes using ICl.}

Reaction of methyl-2-propiolate with ICl. To a solution of methyl-2-propiolate 4 (50 $\mathrm{mg}, 0.60 \mathrm{mmol}, 1.0$ equiv) in dichloroethane $(25 \mathrm{~mL})$ was added iodine monochloride (1.0 M solution in DCM, $0.60 \mathrm{~mL}, 0.60 \mathrm{mmol}, 1$ equiv) and the resulting mixture was stirred for $2 \mathrm{~h}$. The reaction was diluted with $\mathrm{Et}_{2} \mathrm{O}$ and washed sequentially with $\mathrm{NaHSO}_{3}$ ( $20 \%$ wt solution), saturated $\mathrm{NaHCO}_{3}$ and brine. The organic phase was then dried over anhydrous $\mathrm{MgSO}_{4}$, filtered and concentrated in vacuo. The pure product was obtained by flash chromatography eluting with hexanes then $5 \%$ EtOAc in hexanes to give an inseparable mixture of compounds $\mathbf{9 , 1 0 , 6}$, and $\mathbf{7}$ as a pale yellow oil (100 mg, $68 \%$ ).

(10) ${ }^{1} \mathrm{H}$ NMR (400 MHz, $\left.\mathrm{CDCl}_{3}\right) \delta 7.89(\mathrm{~s}, 1 \mathrm{H}), 3.85$ (s, 3H); ${ }^{13} \mathrm{C}$ NMR $(100 \mathrm{MHz}$, $\left.\mathrm{CDCl}_{3}\right) \delta 162.4(\mathrm{C}), 142.6(\mathrm{CH}), 87.7(\mathrm{C}), 53.9\left(\mathrm{CH}_{3}\right) .(\mathbf{6} / 7){ }^{1} \mathrm{H} \mathrm{NMR}\left(400 \mathrm{MHz}, \mathrm{CDCl}_{3}\right)$ $\delta 7.76(\mathrm{~s}, 1 \mathrm{H}), 3.849(\mathrm{~s}, 3 \mathrm{H}) ;{ }^{13} \mathrm{C} \mathrm{NMR}\left(100 \mathrm{MHz}, \mathrm{CDCl}_{3}\right) \delta 162.9(\mathrm{C}), 128.3(\mathrm{C}), 85.5$ (CH), $53.4\left(\mathrm{CH}_{3}\right) ;(6 / 7){ }^{1} \mathrm{H}$ NMR $\left(400 \mathrm{MHz}, \mathrm{CDCl}_{3}\right) \delta 7.35(\mathrm{~s}, 1 \mathrm{H}), 3.85(\mathrm{~s}, 3 \mathrm{H}) ;{ }^{13} \mathrm{C}$ NMR (100 MHz, $\left.\mathrm{CDCl}_{3}\right) \delta 164.0(\mathrm{C}), 97.5(\mathrm{C}), 87.4(\mathrm{CH}), 53.4\left(\mathrm{CH}_{3}\right)$; IR (neat) 1725, $1563 \mathrm{~cm}^{-1}$; MS (EI) $246\left(\mathrm{M}^{+}\right)$. 
Reaction of ethyl 2-butynoate with ICl. Prepared from ethyl 2-butynoate (50 mg, $0.45 \mathrm{mmol}$ ) using a procedure similar to that described above for compound $\mathbf{1 0}$ that provided an inseparable mixture of $\mathbf{1 2}, \mathbf{1 3}, \mathbf{1 4}$, and $\mathbf{1 5}$ as a yellow oil (110 mg, 90\%).

(13) ${ }^{1} \mathrm{H}$ NMR (300 MHz, $\left.\mathrm{CDCl}_{3}\right) \delta 4.27$ (q, $\left.J=7.2 \mathrm{~Hz}, 2 \mathrm{H}\right), 2.54$ (s, 3H), 1.34 (t, $J=$ $7.2 \mathrm{~Hz}, 3 \mathrm{H}) ;{ }^{13} \mathrm{C}$ NMR $\left(75 \mathrm{MHz}, \mathrm{CDCl}_{3}\right) \delta 166.1(\mathrm{C}), 128.3(\mathrm{C}), 84.9(\mathrm{C}), 62.6\left(\mathrm{CH}_{2}\right)$, $38.3\left(\mathrm{CH}_{3}\right), 13.9\left(\mathrm{CH}_{3}\right) .(\mathbf{1 4} / \mathbf{1 5}){ }^{1} \mathrm{H}$ NMR $\left(300 \mathrm{MHz}, \mathrm{CDCl}_{3}\right) \delta 4.36(\mathrm{q}, J=7.2 \mathrm{~Hz}, 2 \mathrm{H})$, $2.66(\mathrm{~s}, 3 \mathrm{H}), 1.35,(\mathrm{t}, J=7.2 \mathrm{~Hz}, 3 \mathrm{H}) ;{ }^{13} \mathrm{C} \mathrm{NMR}\left(75 \mathrm{MHz}, \mathrm{CDCl}_{3}\right) \delta 163.2(\mathrm{C}), 96.9$ (C), $64.6(\mathrm{C}), 60.9\left(\mathrm{CH}_{2}\right), 25.2\left(\mathrm{CH}_{3}\right), 13.7\left(\mathrm{CH}_{3}\right) .(\mathbf{1 4} / \mathbf{1 5}){ }^{1} \mathrm{H} \mathrm{NMR}\left(300 \mathrm{MHz}, \mathrm{CDCl}_{3}\right) \delta 4.18$ (t, $J=7.2 \mathrm{~Hz}, 2 \mathrm{H}), 2.48,(\mathrm{~s}, 3 \mathrm{H}), 1.24(\mathrm{t}, J=7.2 \mathrm{~Hz}, 3 \mathrm{H})$; IR (neat) $1728,1627 \mathrm{~cm}^{-1}$; MS (EI) $274\left(\mathrm{M}^{+}\right)$.

Reaction of 3-cyclohexylpropiolate with ICl. Prepared from ethyl 3cyclohexylpropiolate $(50 \mathrm{mg}, 0.28 \mathrm{mmol})$ using a procedure similar to that described above for compound $\mathbf{1 0}$ that provided an inseparable mixture of $\mathbf{2 0}$ and $\mathbf{2 1}$ as a yellow oil (95 mg, 99\%). (21) ${ }^{1} \mathrm{H}$ NMR (300 MHz, $\left.\mathrm{CDCl}_{3}\right) \delta 4.29$ (q, $\left.J=7.2 \mathrm{~Hz}, 2 \mathrm{H}\right), 2.96$ (tt, $J=$ 11.1, $3.3 \mathrm{~Hz}, 1 \mathrm{H}), 2.20-1.16(\mathrm{~m}, 10 \mathrm{H}), 1.33$ (t, $J=7.2 \mathrm{~Hz}, 3 \mathrm{H}) ;{ }^{13} \mathrm{C}$ NMR $(75 \mathrm{MHz}$, $\left.\mathrm{CDCl}_{3}\right) \delta 165.6(\mathrm{C}), 143.0(\mathrm{C}), 68.5(\mathrm{C}), 62.9\left(\mathrm{CH}_{2}\right), 52.0(\mathrm{CH}), 32.8\left(\mathrm{CH}_{2}\right), 26.4\left(\mathrm{CH}_{2}\right)$, $23.2\left(\mathrm{CH}_{2}\right), 14.3\left(\mathrm{CH}_{3}\right)$; IR (neat) 1732, $1611 \mathrm{~cm}^{-1}$; $\mathrm{MS}(\mathrm{EI}) 342\left(\mathrm{M}^{+}\right)$.

Reaction of ethyl phenylpropiolate with ICI. Prepared from ethyl phenylpropiolate (50 $\mathrm{mg}, 0.29 \mathrm{mmol}$ ) using a procedure similar to that described above for compound $\mathbf{1 0}$ that provided an inseparable mixture of 23 and 24 as a yellow oil (89 mg, 93\%). (24) ${ }^{1} \mathrm{H}$ $\operatorname{NMR}\left(500 \mathrm{MHz}, \mathrm{CDCl}_{3}\right) \delta 7.47-7.26(\mathrm{~m}, 5 \mathrm{H}), 3.99(\mathrm{q}, J=7.0 \mathrm{~Hz}, 2 \mathrm{H}), 0.94(\mathrm{t}, J=7.0$ $\mathrm{Hz}, 3 \mathrm{H}) ;{ }^{13} \mathrm{C}$ NMR (125 MHz, $\left.\mathrm{CDCl}_{3}\right) \delta 165.6(\mathrm{C}), 149.0(\mathrm{C}), 138.6(\mathrm{C}), 134.7(\mathrm{CH})$, 
$129.8(\mathrm{CH}), 127.9(\mathrm{CH}), 81.2(\mathrm{C}), 62.5\left(\mathrm{CH}_{2}\right), 13.4\left(\mathrm{CH}_{3}\right)$; IR (neat) 1728, $1615 \mathrm{~cm}^{-1}$; MS (EI) $336\left(\mathrm{M}^{+}\right)$.

Prepared from 1,4-bis(benzyloxy)but-2-yne $(50 \mathrm{mg}, 0.19 \mathrm{mmol})$ using a procedure similar to that described above for compound $\mathbf{1 0}$ that provided an inseparable mixture of compounds 30 and 31 as a yellow oil (81 mg, $99 \%$ ). (31) ${ }^{1} \mathrm{H}$ NMR (400 MHz, $\mathrm{CDCl}_{3}$ ) $\delta 7.49-7.29(\mathrm{~m}, 10 \mathrm{H}), 4.57(\mathrm{~s}, 2 \mathrm{H}), 4.54(\mathrm{~s}, 2 \mathrm{H}), 4.51(\mathrm{~s}, 2 \mathrm{H}), 4.47(\mathrm{~s}, 2 \mathrm{H}) ;{ }^{13} \mathrm{C} \mathrm{NMR}$ $\left(100 \mathrm{MHz}, \mathrm{CDCl}_{3}\right) \delta 131.3(\mathrm{C}), 128.6(\mathrm{C}), 128.5(\mathrm{C}), 128.4(\mathrm{CH}), 127.92(\mathrm{CH}), 127.88$ $(\mathrm{CH}), 100.8(\mathrm{C}), 81.5\left(\mathrm{CH}_{2}\right), 76.3\left(\mathrm{CH}_{2}\right), 73.1\left(\mathrm{CH}_{2}\right), 71.9\left(\mathrm{CH}_{2}\right)$; IR (neat) 1461, 1376 $\mathrm{cm}^{-1}$; MS (EI) $393\left(\mathrm{M}^{+}-\mathrm{Cl}\right), 301\left(\mathrm{M}^{+}-\mathrm{I}\right)$.

Reaction of alloxybenzene with ICl. Prepared from allyloxybenzene $(50 \mathrm{mg}, 0.37$ mmol) using a procedure similar to that described above for compound $\mathbf{1 0}$ that provided an inseparable mixture of compounds $\mathbf{3 3}$ and $\mathbf{3 4}$ as a yellow oil $(89.8 \mathrm{mg}, 82 \%)$. (34) ${ }^{1} \mathrm{H}$ NMR (400 MHz, $\left.\mathrm{CDCl}_{3}\right) \delta 7.37-7.33(\mathrm{~m}, 2 \mathrm{H}), 7.06-7.02(\mathrm{~m}, 1 \mathrm{H}), 6.99-6.96(\mathrm{~m}$, 2H), $4.54-4.29(\mathrm{~m}, 1 \mathrm{H}), 4.07(\mathrm{~d}, J=6.8 \mathrm{~Hz}, 2 \mathrm{H}), 3.74(\mathrm{dd}, J=10.7,6.4 \mathrm{~Hz}, 1 \mathrm{H}), 3.67$ (dd, $J=10.7,4.5 \mathrm{~Hz}, 1 \mathrm{H}) ;{ }^{13} \mathrm{C}$ NMR $\left(100 \mathrm{MHz}, \mathrm{CDCl}_{3}\right) \delta 157.8(\mathrm{C}), 129.3(\mathrm{CH}), 121.6$ (CH), $114.7(\mathrm{CH}), 70.2\left(\mathrm{CH}_{2}\right), 54.1(\mathrm{CH}), 6.7\left(\mathrm{CH}_{2}\right)$; IR (neat) 1594, $1500 \mathrm{~cm}^{-1}$; MS (EI) $296\left(\mathrm{M}^{+}\right)$.

Reaction of pent-4-enyl benzoate with ICl. Prepared from pent-4-enyl benzoate ${ }^{8}$ (50 $\mathrm{mg}, 0.26 \mathrm{mmol}$ ) using a procedure similar to that described above for compound $\mathbf{1 0}$ that provided an inseparable mixture of compounds $\mathbf{3 6}$ and $\mathbf{3 7}$ as a colorless oil (85 $\mathrm{mg}, 92 \%)$. (37) ${ }^{1} \mathrm{H}$ NMR (400 MHz, acetone-d 6 ) $\delta 8.06(\mathrm{~d}, J=10.4 \mathrm{~Hz}, 2 \mathrm{H}), 7.57(\mathrm{dd}, J=10.0,10.0$ Hz), 7.44 (dd, $J=10.4,10.4 \mathrm{~Hz}), 4.37$ (t, $J=8.0 \mathrm{~Hz}, 2 \mathrm{H}), 4.35-4.23(\mathrm{~m}, 1 \mathrm{H}), 4.09-$ $3.86(\mathrm{dd}, J=17.2,5.6 \mathrm{~Hz}, 1 \mathrm{H}), 3.83(\mathrm{dd}, J=14.4,14.4 \mathrm{~Hz}, 1 \mathrm{H}), 2.33-1.86(\mathrm{~m}, 4 \mathrm{H}) ;{ }^{13} \mathrm{C}$ 
NMR (100 MHz, acetone-d $\left.{ }_{6}\right) \delta 166.5(\mathrm{C}), 133.0(\mathrm{CH}), 130.1(\mathrm{C}), 129.5(\mathrm{CH}), 128.4$ $(\mathrm{CH}), 63.7\left(\mathrm{CH}_{2}\right), 33.9(\mathrm{CH}), 33.1\left(\mathrm{CH}_{2}\right), 28.2\left(\mathrm{CH}_{2}\right), 10.3\left(\mathrm{CH}_{2}\right)$; IR (neat) 1718, 1615 $\mathrm{cm}^{-1}$; MS (EI) $225\left(\mathrm{M}^{+}-\mathrm{I}\right), 317\left(\mathrm{M}^{+}-\mathrm{Cl}\right)$.

2-(2-chloro-3-iodopropyl)phenol (40). Prepared from 2-allylphenol ${ }^{6}$ (50 mg, 0.37 mmol) using a procedure similar to that described above for compound $\mathbf{1 0}$ that provided compound 40 as a yellow oil (109 mg, 99\%). ${ }^{1} \mathrm{H}$ NMR $\left(400 \mathrm{MHz}, \mathrm{CDCl}_{3}\right) \delta 7.17-7.11$ (m, 2H), $6.87(\mathrm{ddd}, J=8.0,7.6,0.8 \mathrm{~Hz}, 1 \mathrm{H}), 6.78(\mathrm{~d}, J=8.0 \mathrm{~Hz}), 4.92-4.85(\mathrm{~m}, 1 \mathrm{H})$, $3.45\left(\mathrm{dd}, J_{\mathrm{AB}}=8.8, J_{\mathrm{AX}}=6.4 \mathrm{~Hz}, 1 \mathrm{H}\right), 3.34\left(\mathrm{dd}, J_{\mathrm{AB}}=8.8, J_{\mathrm{BX}}=6.1 \mathrm{~Hz}, 1 \mathrm{H}\right), 3.40(\mathrm{dd}, J$ $=15.7,8.9 \mathrm{~Hz}, 1 \mathrm{H}), 3.05(\mathrm{dd}, J=15.7,6.4 \mathrm{~Hz}) ;{ }^{13} \mathrm{C} \mathrm{NMR}\left(100 \mathrm{MHz}, \mathrm{CDCl}_{3}\right) \delta 159.1$ (C), $128.2(\mathrm{CH}), 125.7(\mathrm{C}), 125.0(\mathrm{CH}), 120.8(\mathrm{CH}), 109.6(\mathrm{CH}), 81.6(\mathrm{CH}), 36.1\left(\mathrm{CH}_{2}\right)$, $8.9\left(\mathrm{CH}_{2}\right)$; IR (neat) 1506, $1479 \mathrm{~cm}^{-1}$; HRMS calc'd for $\mathrm{C}_{9} \mathrm{H}_{10} \mathrm{ClIO}\left(\mathrm{M}^{+}\right)$295.9465, found 295.9467.

Reaction of 5-decene with ICl. ${ }^{9}$ Prepared from 5-decene $(0.135 \mathrm{~mL}, 0.71 \mathrm{mmol})$ using a procedure similar to that described above for compound $\mathbf{7}$ that provided compound $\mathbf{4 3}$ as an inseparable mixture of 5,6-dichlorodecane (42) and 5-chloro-6-iododecane (43) as a colorless oil (142 mg, 66\%). ${ }^{1} \mathrm{H}$ NMR (400 MHz, $\left.\mathrm{CDCl}_{3}\right) \delta 4.26-4.21(\mathrm{~m}, 1 \mathrm{H}), 3.93-$ $3.88(\mathrm{~m}, 1 \mathrm{H}), 2.11-2.03(\mathrm{~m}, 1 \mathrm{H}), 1.98-1.80(\mathrm{~m}, 3 \mathrm{H}), 1.62-1.52(\mathrm{~m}, 2 \mathrm{H}), 1.46-1.27$ (m, 6H), $0.93(\mathrm{t}, J=7.3 \mathrm{~Hz}, 3 \mathrm{H}) ;{ }^{13} \mathrm{C}$ NMR $\left(100 \mathrm{MHz}\right.$, acetone-d $\left.\mathrm{d}_{6}\right) \delta 66.9(\mathrm{CH}), 42.5$ $(\mathrm{CH}), 37.5\left(\mathrm{CH}_{2}\right), 36.8(\mathrm{CH}), 31.3\left(\mathrm{CH}_{2}\right), 28.2\left(\mathrm{CH}_{2}\right), 22.1\left(\mathrm{CH}_{2}\right), 21.9\left(\mathrm{CH}_{2}\right), 13.9$ $\left(\mathrm{CH}_{3}\right)$; IR (neat) 2957, 2931, $1465 \mathrm{~cm}^{-1}$; MS (EI) $302\left(\mathrm{M}^{+}\right)$; HRMS calc'd for $\mathrm{C}_{10} \mathrm{H}_{20} \mathrm{ClI}$ $(\mathrm{M}+)$ 302.0298, found 302.0218.

Reaction of ((3-methylbut-3-enyloxy)methyl)benzene with ICl. Prepared from ((3methylbut-3-enyloxy)methyl)benzene ${ }^{10}(50 \mathrm{mg}, 0.28 \mathrm{mmol})$ using a procedure similar to 
that described above for compound $\mathbf{1 0}$ that provided an inseparable mixture of compounds 45 and 46 as a colorless oil (12 mg, 14\%). ${ }^{1} \mathrm{H}$ NMR (400 $\left.\mathrm{MHz}, \mathrm{CDCl}_{3}\right) \delta$ $7.36-7.36(\mathrm{~m}, 5 \mathrm{H}), 4.51(\mathrm{~s}, 2 \mathrm{H}), 3.81-3.60(\mathrm{~m}, 4 \mathrm{H}), 2.29-2.15(\mathrm{~m}, 2 \mathrm{H}), 1.66(\mathrm{~s}, 3 \mathrm{H})$

${ }^{13} \mathrm{C}$ NMR $\left(100 \mathrm{MHz}, \mathrm{CDCl}_{3}\right) \delta 138.1(\mathrm{C}), 128.4(\mathrm{CH}), 127.6(\mathrm{CH}), 127.5(\mathrm{CH}), 73.1$ $\left(\mathrm{CH}_{2}\right), 70.2(\mathrm{C}), 66.7\left(\mathrm{CH}_{2}\right), 41.2\left(\mathrm{CH}_{2}\right), 28.6\left(\mathrm{CH}_{3}\right), 18.9\left(\mathrm{CH}_{2}\right)$; IR (neat) 2925,1273 $\mathrm{cm}^{-1} ; \mathrm{MS}(\mathrm{EI}) 338\left(\mathrm{M}^{+}\right)$.

Reaction of $\boldsymbol{n}$-butyl acrylate with ICl. Prepared from $n$-butyl acrylate (50 mg, 0.39 mmol) using a procedure similar to that described above for compound $\mathbf{1 0}$ that provided an inseparable mixture of compounds 48 and 49 as a yellow oil (67 mg, $61 \%)^{7}$

\section{Summary of variable $\mathrm{Bu}_{4} \mathrm{NI}$ amount control reactions:}

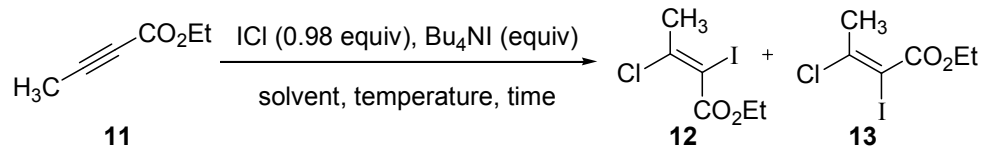

General procedure: To a solution of alkyne 11 (50 $\mathrm{mg}, 0.45 \mathrm{mmol}, 1$ equiv) in DCE (4.0 mL) was added $\mathrm{Bu}_{4} \mathrm{NI}(41 \mathrm{mg}, 0.11 \mathrm{mmol}, 0.25$ equiv). To this was added $\mathrm{ICl}(1 \mathrm{M}$ solution in DCM, $0.44 \mathrm{~mL}, 0.44 \mathrm{mmol}, 0.98$ equiv). The reaction was stirred for 2 hours, upon which time the solution was diluted with $\mathrm{Et}_{2} \mathrm{O}$ and washed sequentially with $\mathrm{NaHSO}_{3}\left(20 \%\right.$ solution), a saturated solution of $\mathrm{NaHCO}_{3}$ and brine. The organic layer was dried over anhydrous $\mathrm{MgSO}_{4}$, filtered and concentrated to give a mixture of compounds 12:13 (28 mg, $23 \%$ combined yield).

\begin{tabular}{cccccc} 
Equiv Bu $\mathbf{H}_{\mathbf{4}} \mathbf{N I}$ & Temperature & Time & Solvent & Yield (\%) & 12:13 Ratio \\
\hline 0.25 & $\mathrm{rt}$ & $2 \mathrm{~h}$ & $\mathrm{DCE}$ & 23 & $19: 1$ \\
0.50 & $\mathrm{rt}$ & $2 \mathrm{~h}$ & $\mathrm{DCE}$ & 6 & $19: 1$ \\
1.05 & $\mathrm{rt}$ & $2 \mathrm{~h}$ & $\mathrm{DCE}$ & trace & - \\
3.00 & $\mathrm{rt}$ & $2 \mathrm{~h}$ & $\mathrm{DCE}$ & no reaction & - \\
3.00 & reflux & $18 \mathrm{~h}$ & $\mathrm{DCE}$ & 30 & 12 exclusively \\
3.00 & reflux & $18 \mathrm{~h}$ & benzene & 2 & 12 exclusively
\end{tabular}




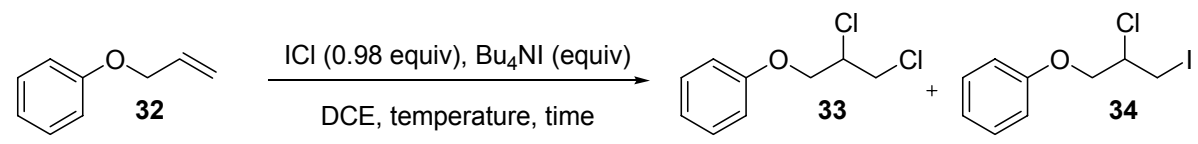

\begin{tabular}{cccccc} 
Equiv Bu $\mathbf{H}_{\mathbf{N}} \mathbf{N I}$ & Temperature & Time & Solvent & Yield (\%) & $\mathbf{3 3 : 3 4}$ \\
\hline 0.25 & $\mathrm{rt}$ & $5 \mathrm{~h}$ & DCE & 62 & $1: 1$ \\
0.50 & $\mathrm{rt}$ & $5 \mathrm{~h}$ & DCE & 11 & $1.2: 1$ \\
1.05 & $\mathrm{rt}$ & $5 \mathrm{~h}$ & DCE & 6 & $1: 1$ \\
3.00 & $\mathrm{rt}$ & $5 \mathrm{~h}$ & DCE & trace & $1: 1$ \\
3.00 & reflux & $18 \mathrm{~h}$ & DCE & 32 & $\mathbf{3 3}$ exclusively \\
3.00 & reflux & $18 \mathrm{~h}$ & benzene & 9 & $\mathbf{3 3}$ exclusively
\end{tabular}
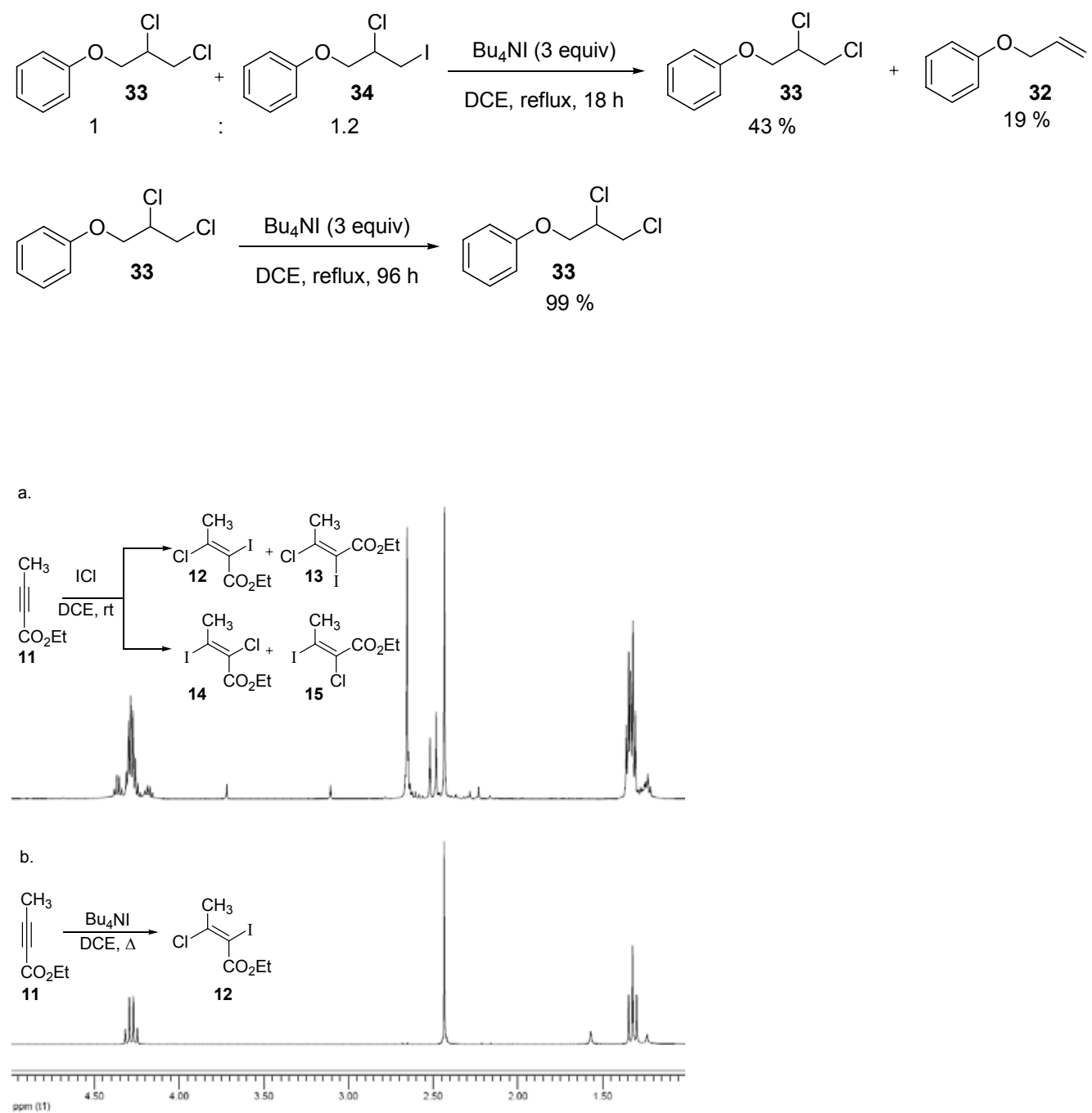

Figure 1. Comparison of the crude products obtained from the reaction of $\mathbf{1 1}$ with $\mathrm{ICl}$ (1a) and with $\mathrm{Bu}_{4} \mathrm{NI} / \mathrm{DCE}(1 \mathrm{~b})$. 
NMR Spectra 

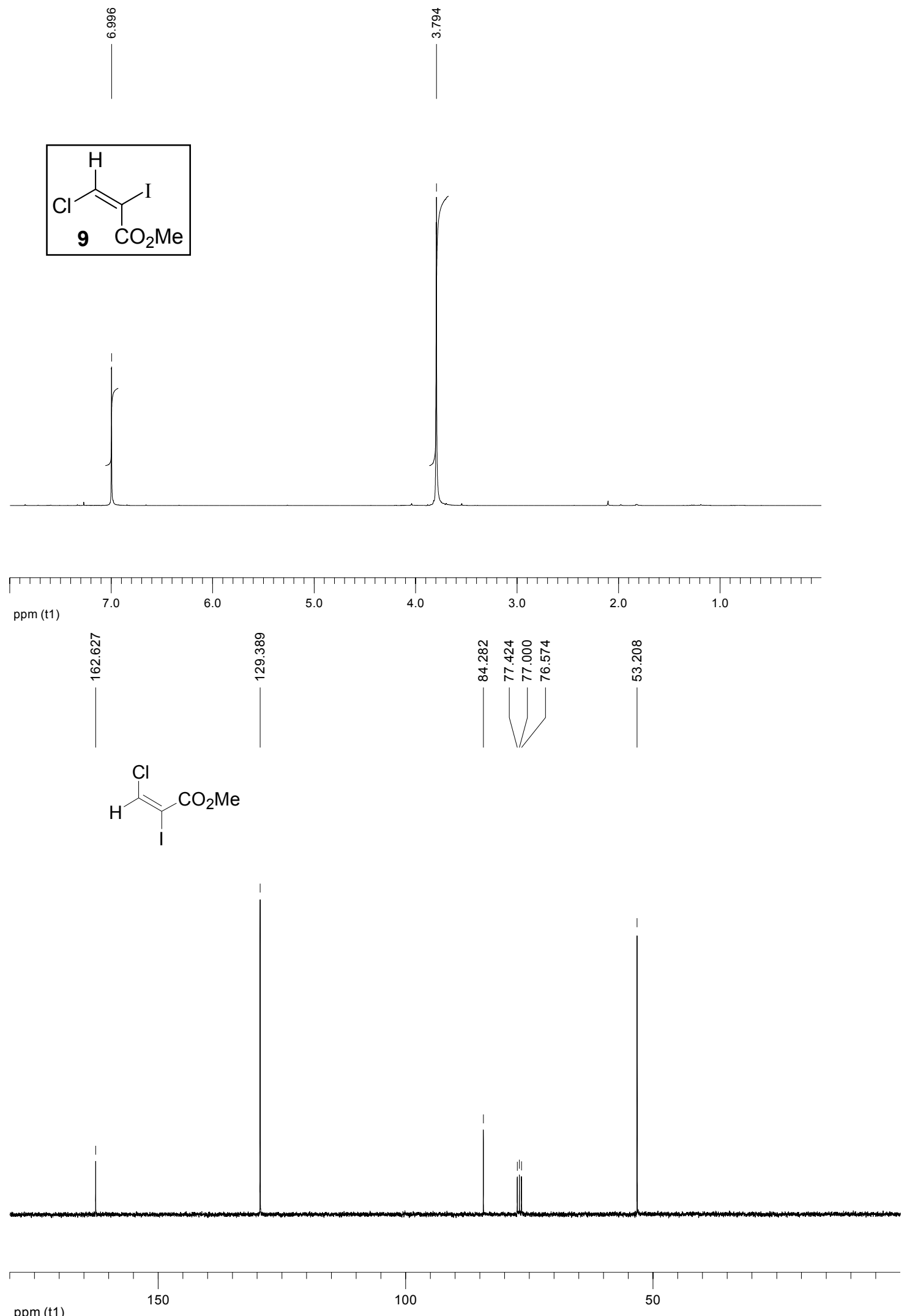

ppm (t1) 

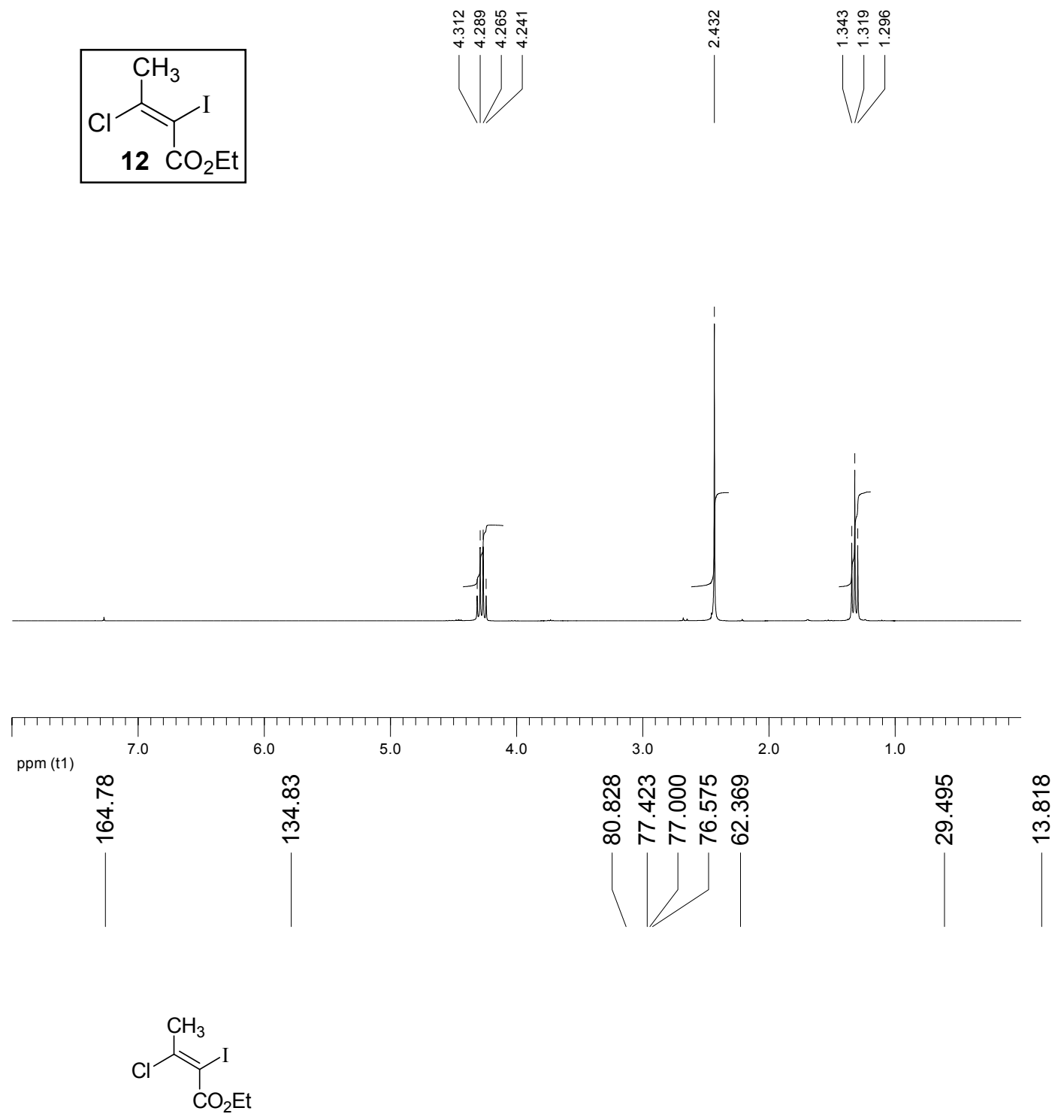

12

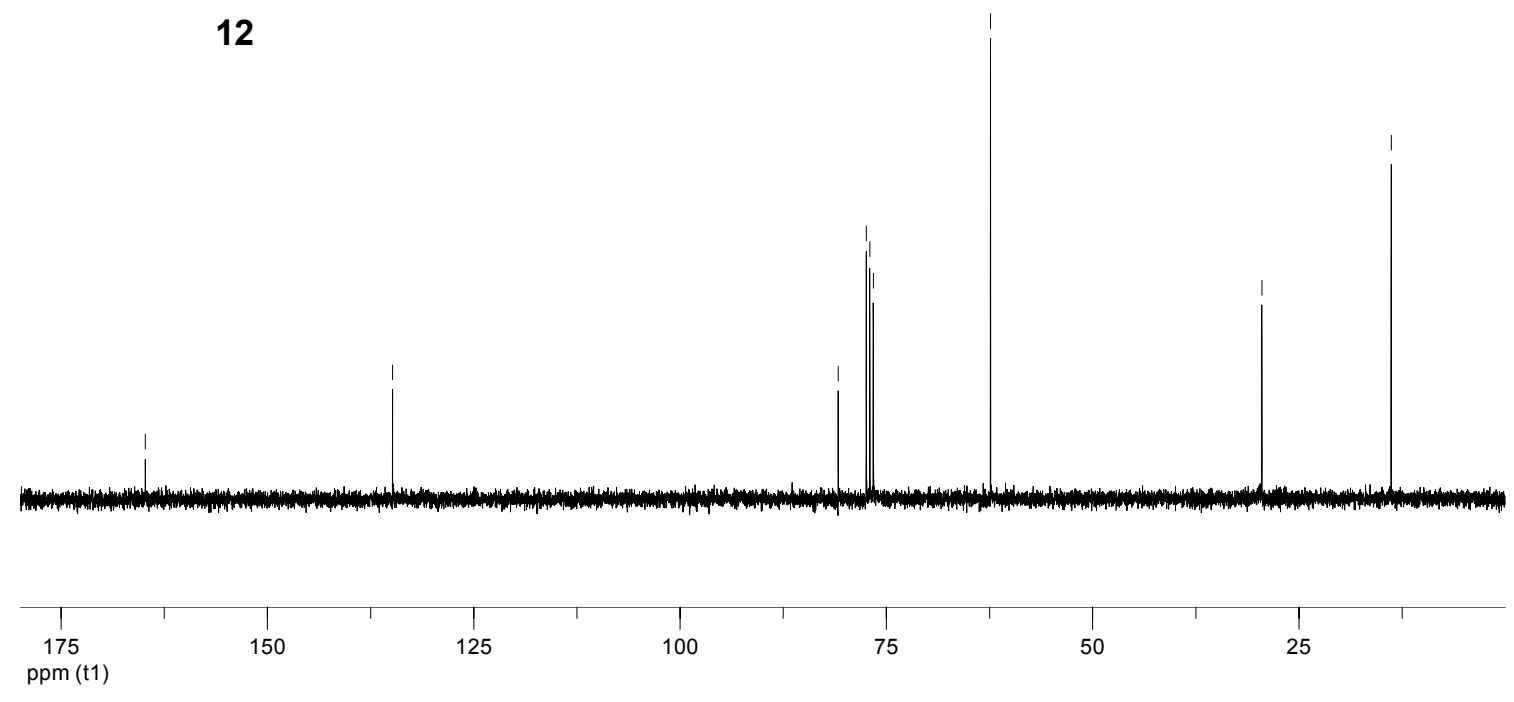



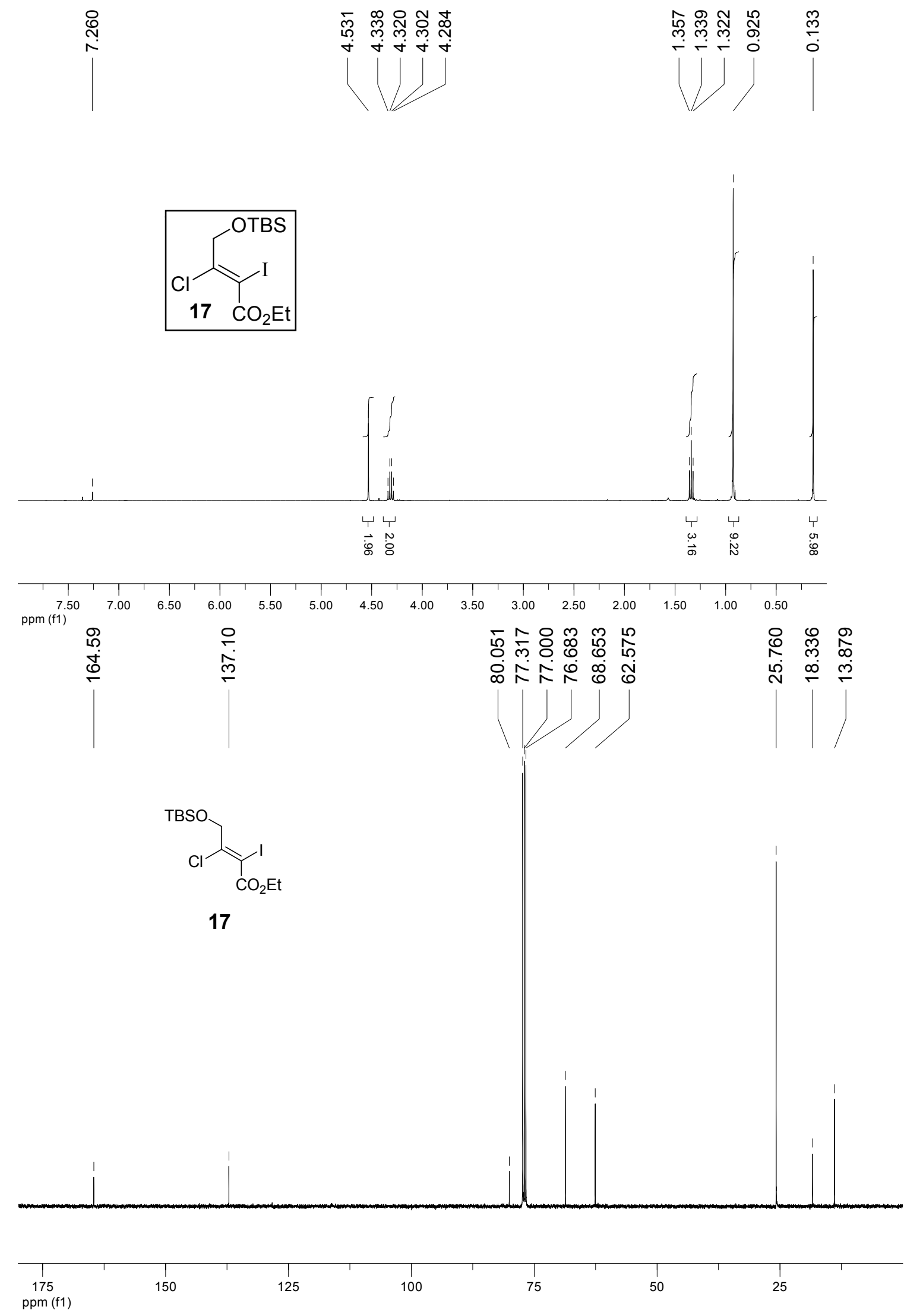

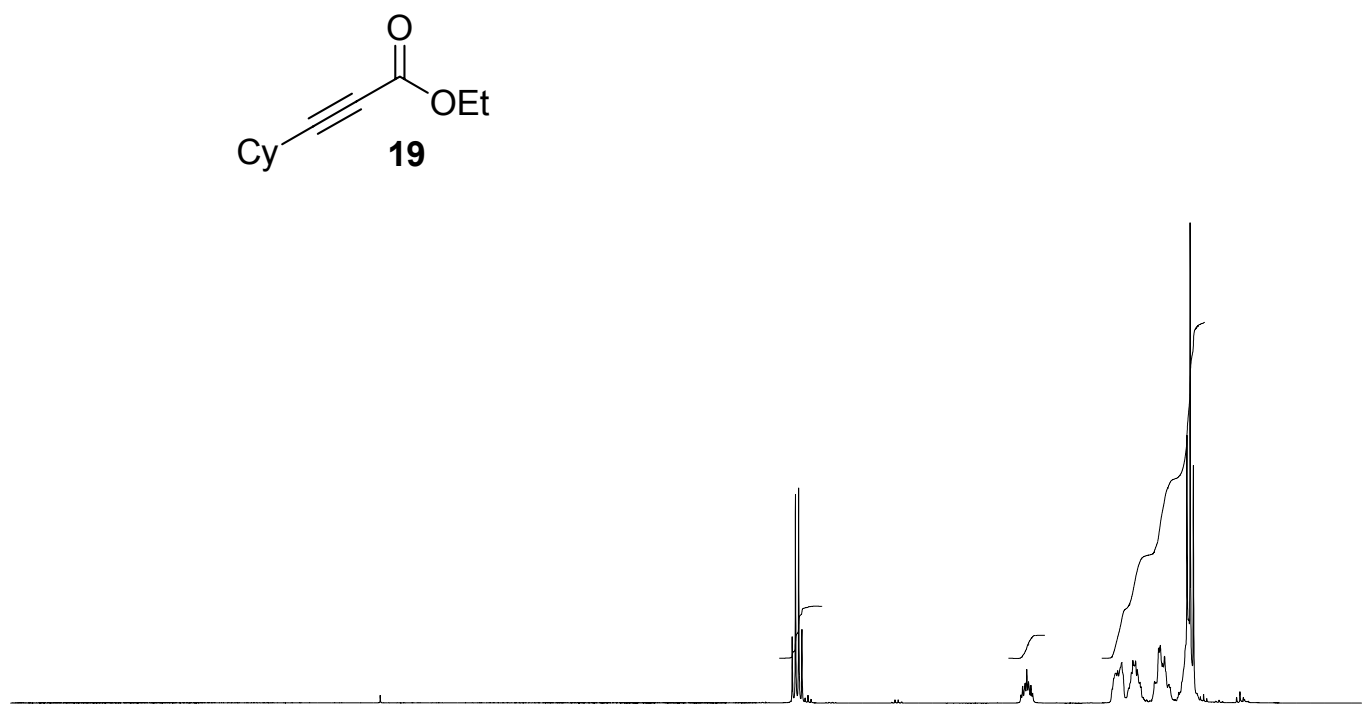

\begin{tabular}{|c|c|c|c|c|c|c|c|c|c|c|c|c|c|c|c|c|c|c|}
\hline . & 1 & $T$ & $T$ & $T$ & $T$ & $T$ & 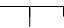 & $T$ & $T$ & $\mid$ & 1 & $\mid$ & 1 & 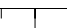 & ] & 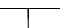 & 1 & $T$ \\
\hline $\begin{array}{r}9.50 \\
\text { ppm (f1) }\end{array}$ & 9.00 & 8.50 & 8.00 & 7.50 & 7.00 & 6.50 & 6.00 & 5.50 & 5.00 & 4.50 & 4.00 & 3.50 & 3.00 & 2.50 & 2.00 & 1.50 & 1.00 & 0.50 \\
\hline
\end{tabular}
ppm (f1)

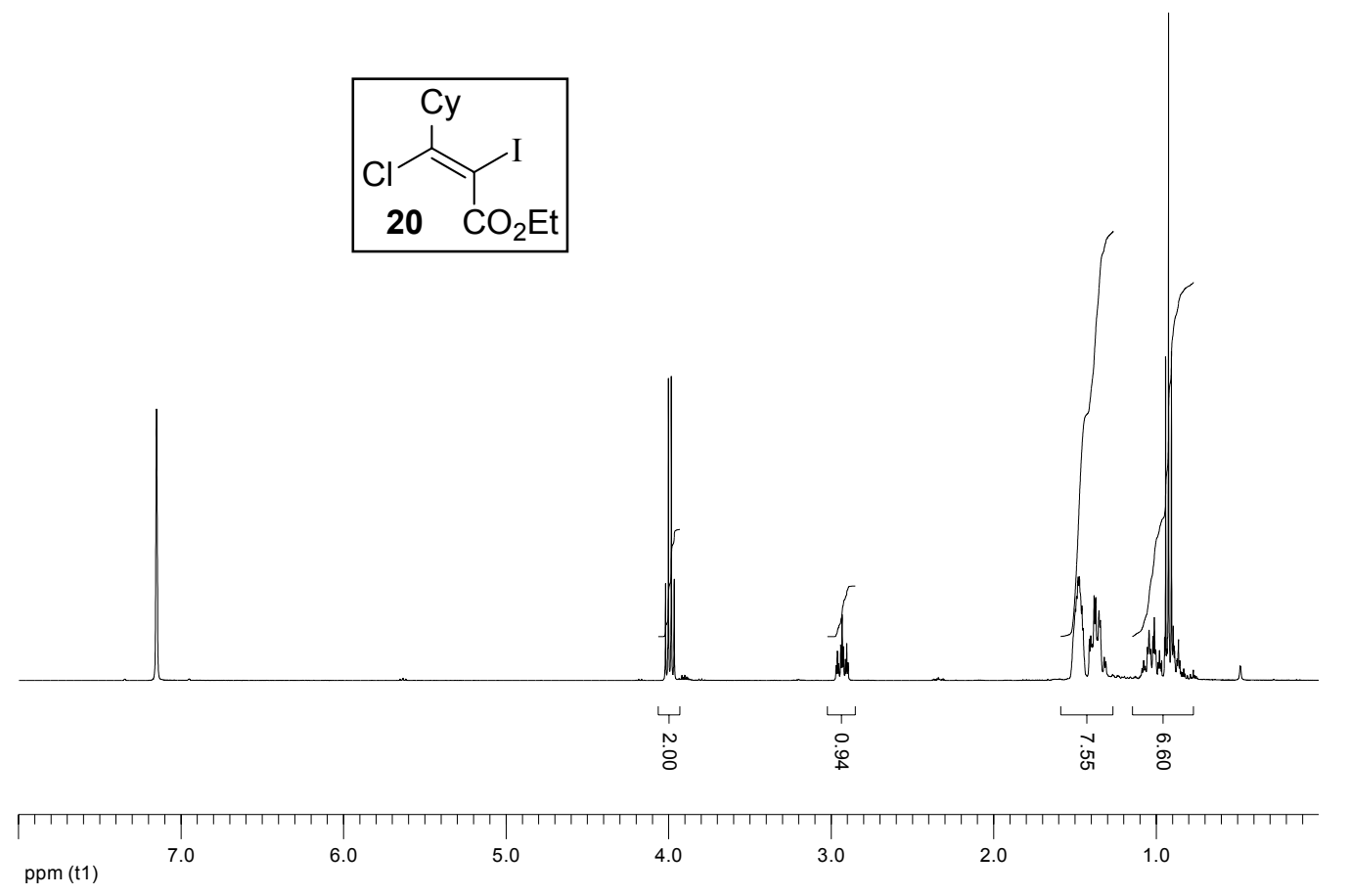




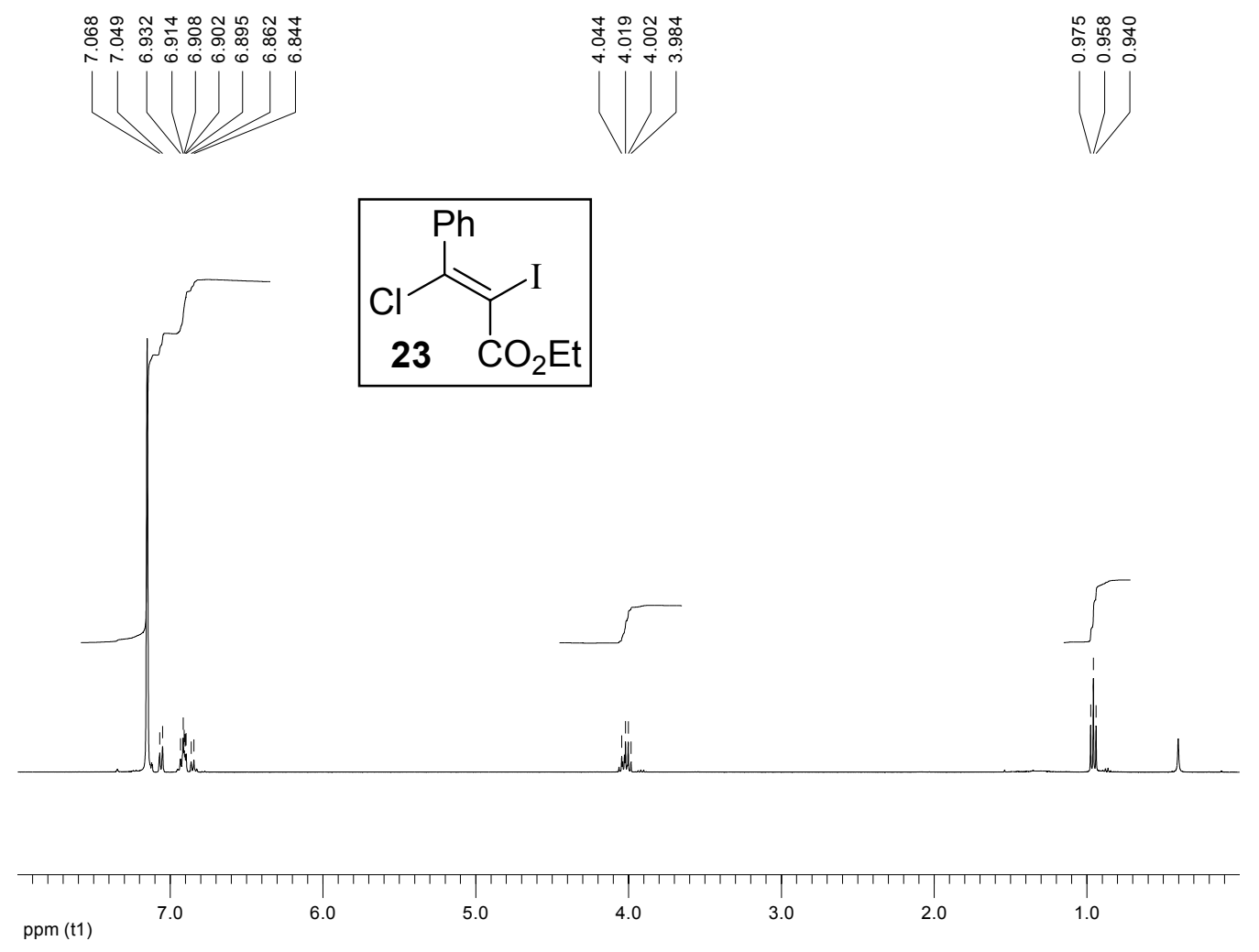




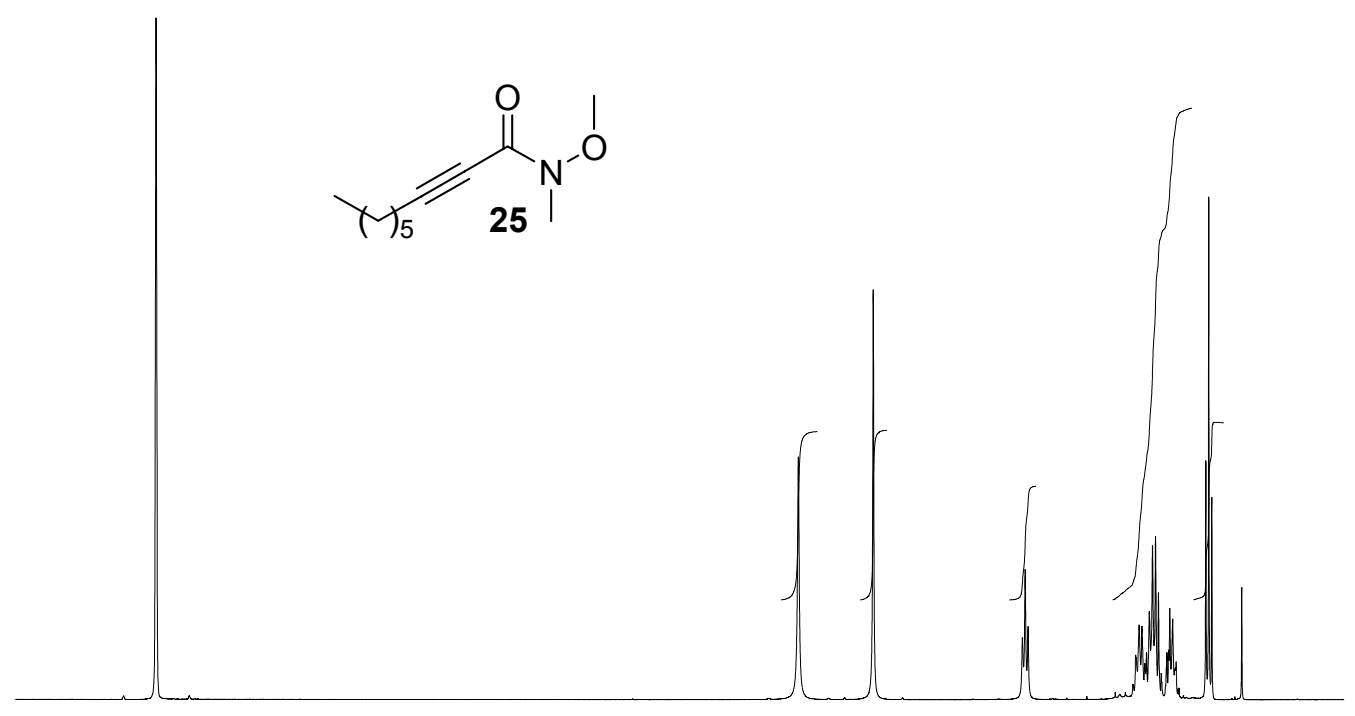

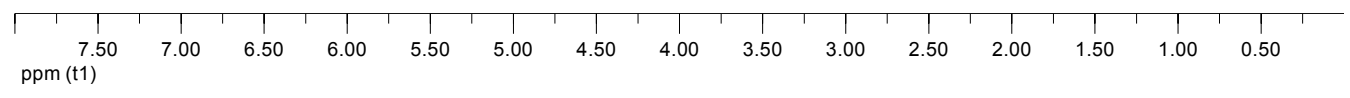

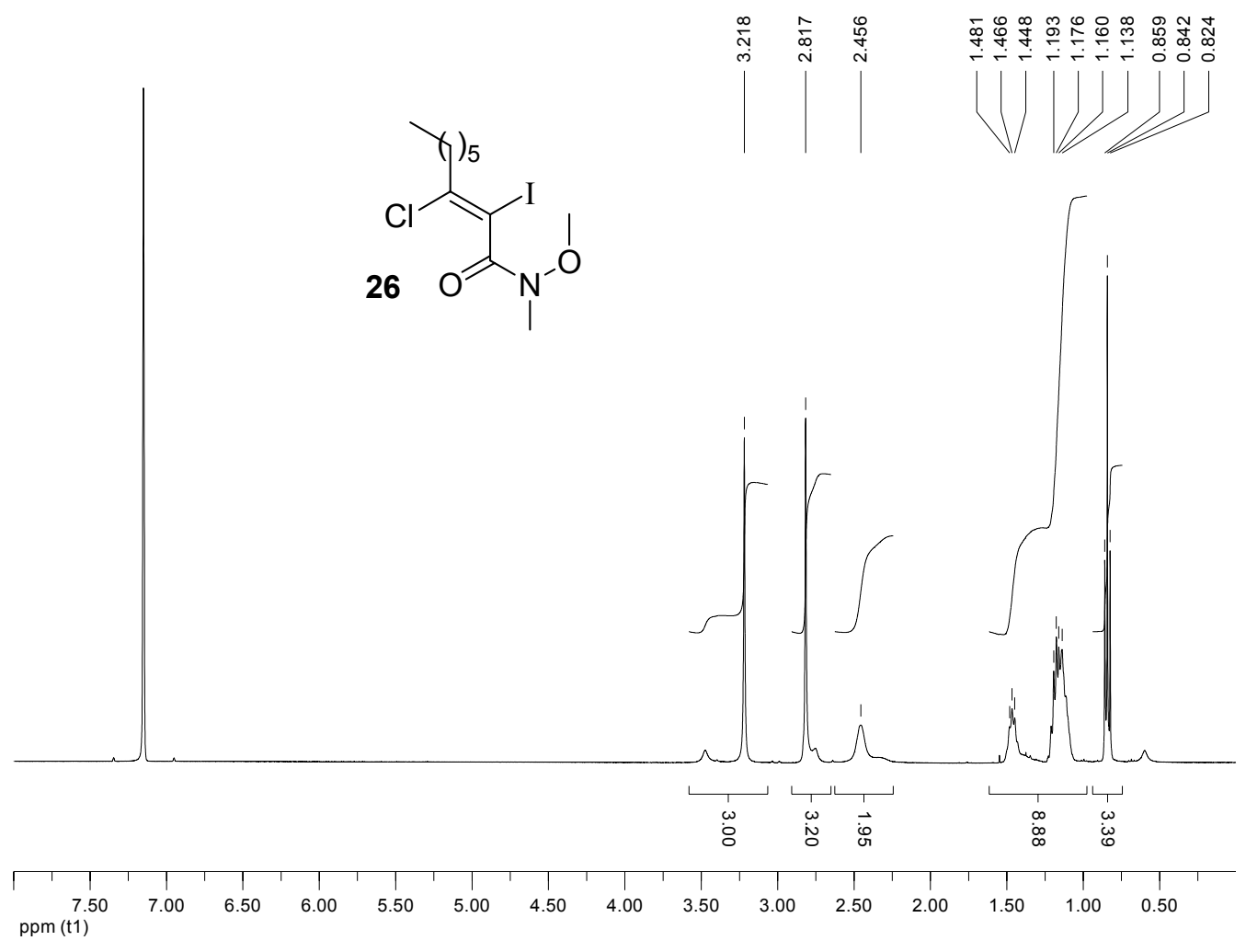




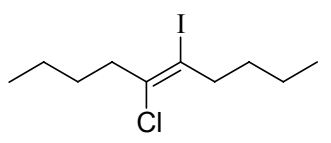

28
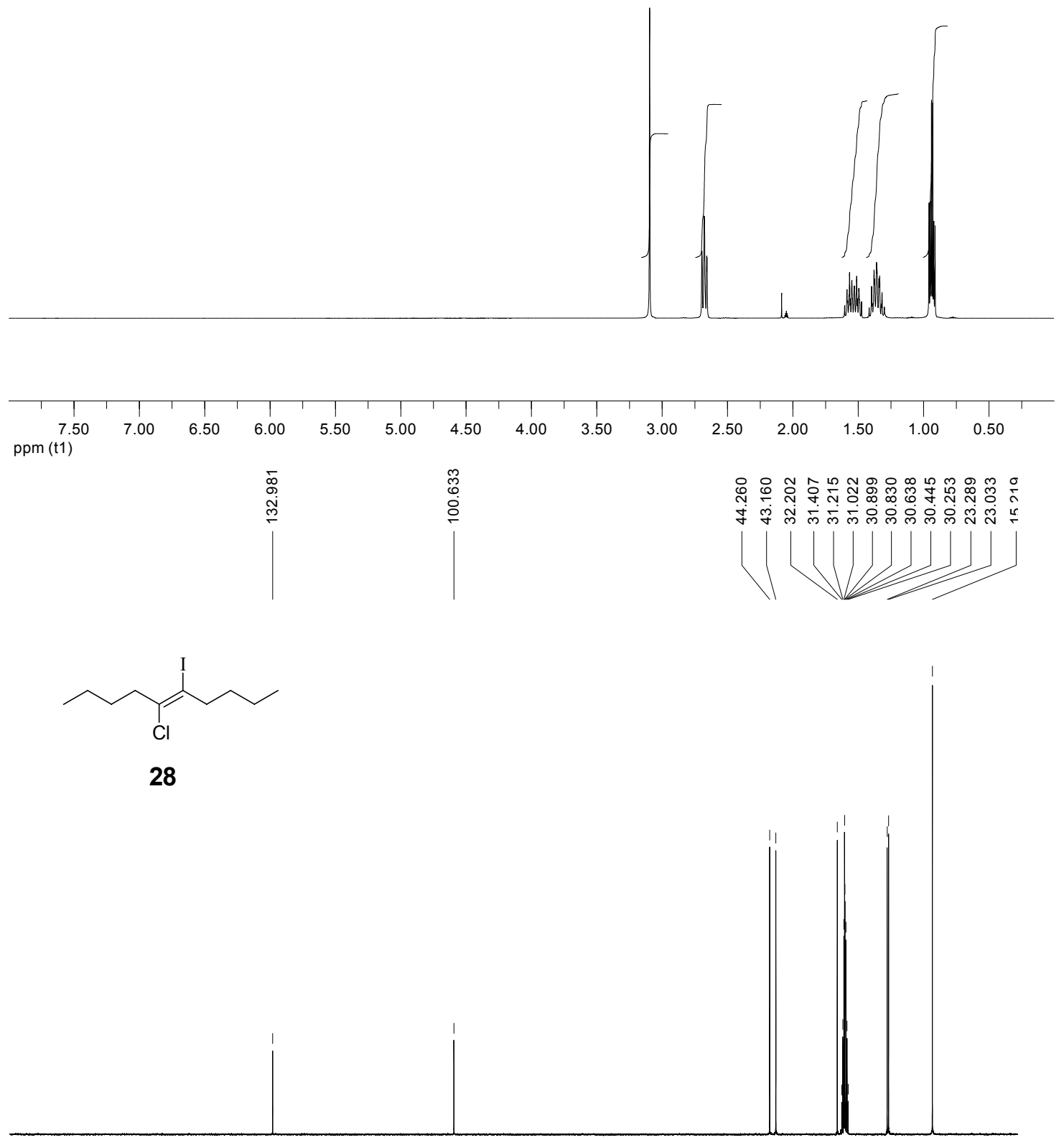

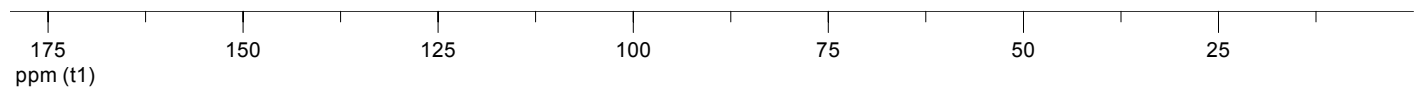



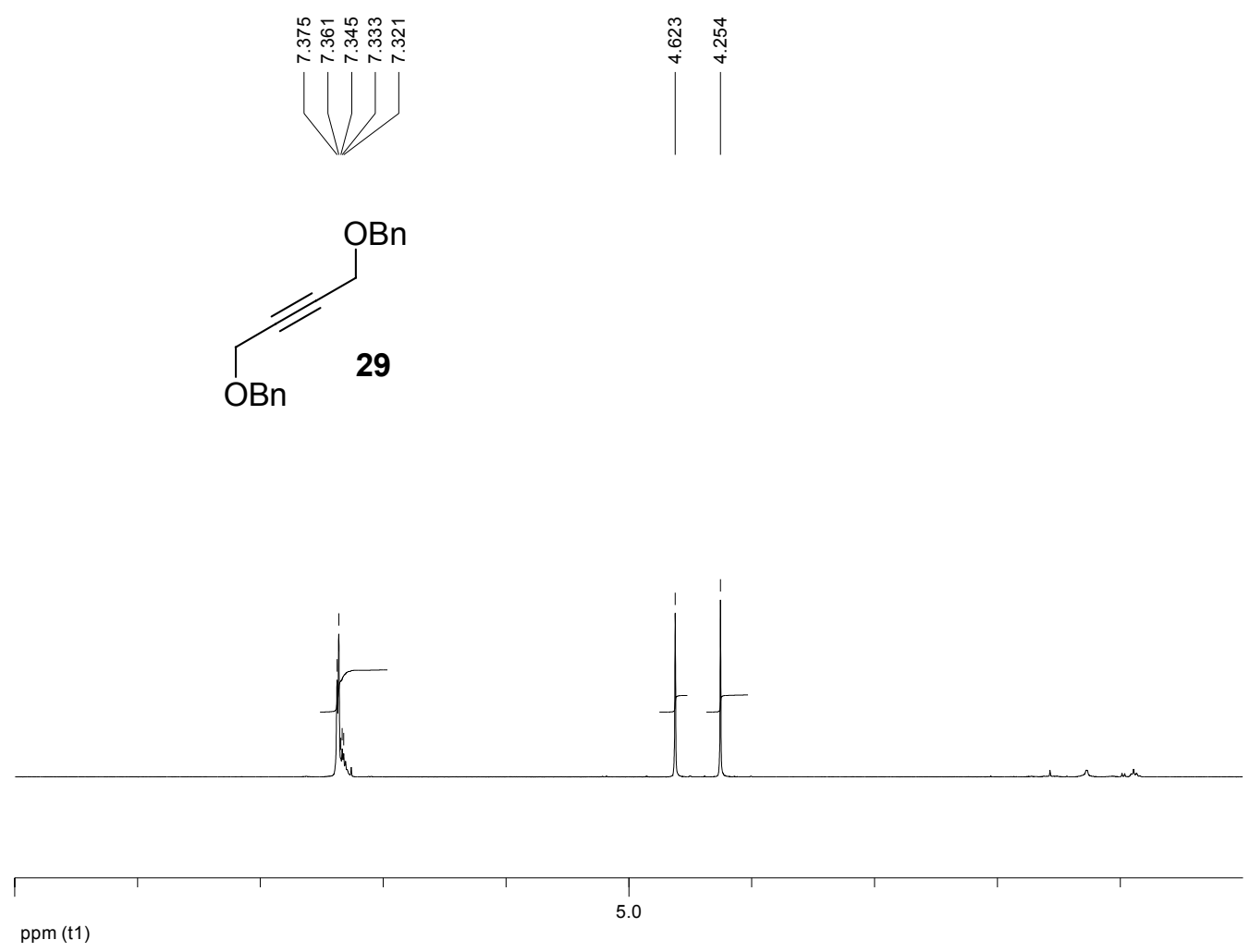

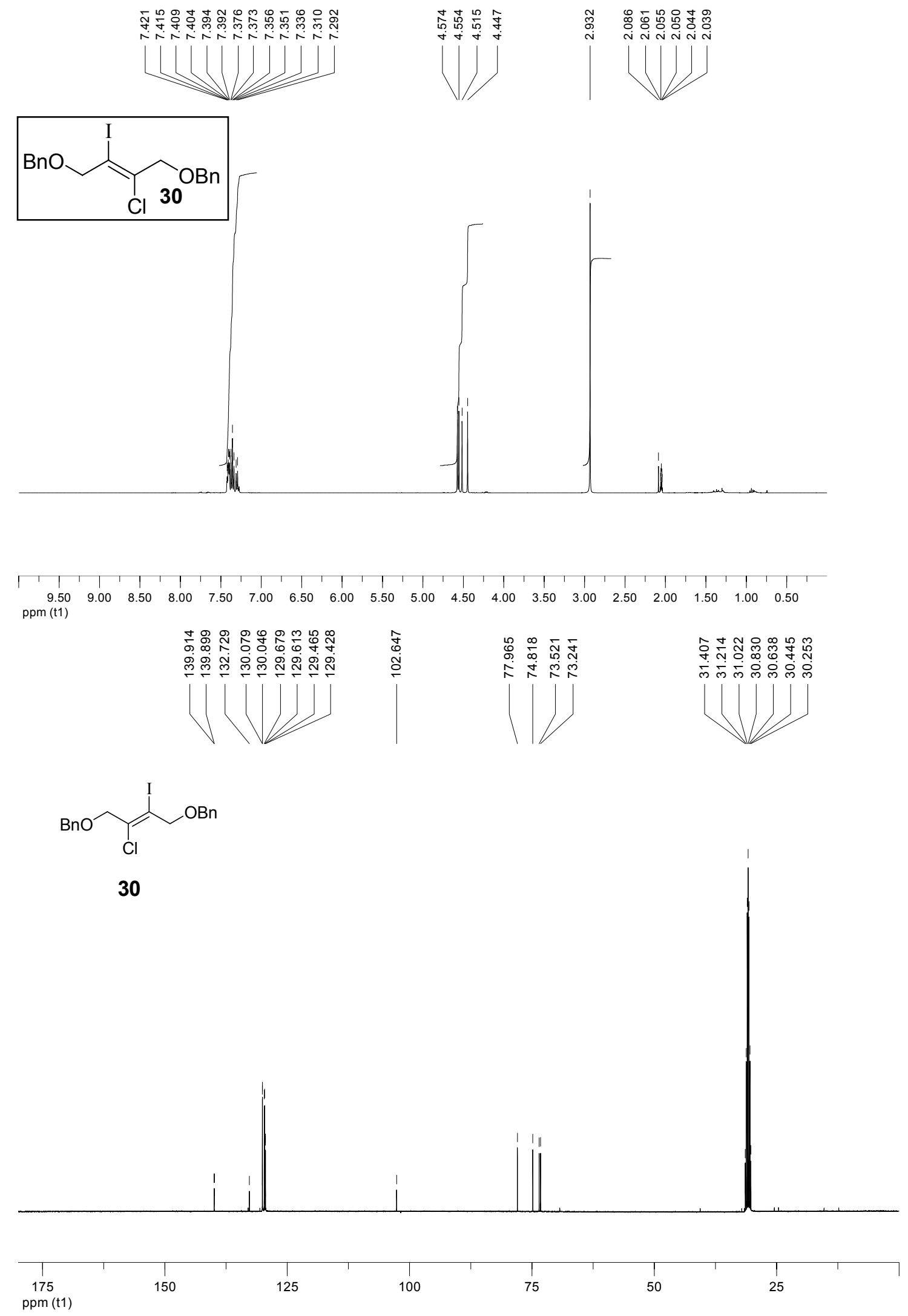


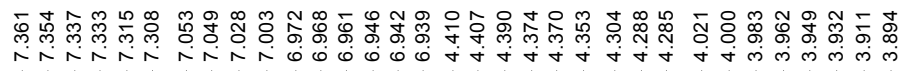<smiles>ClCC(Cl)COc1ccccc1</smiles>

33
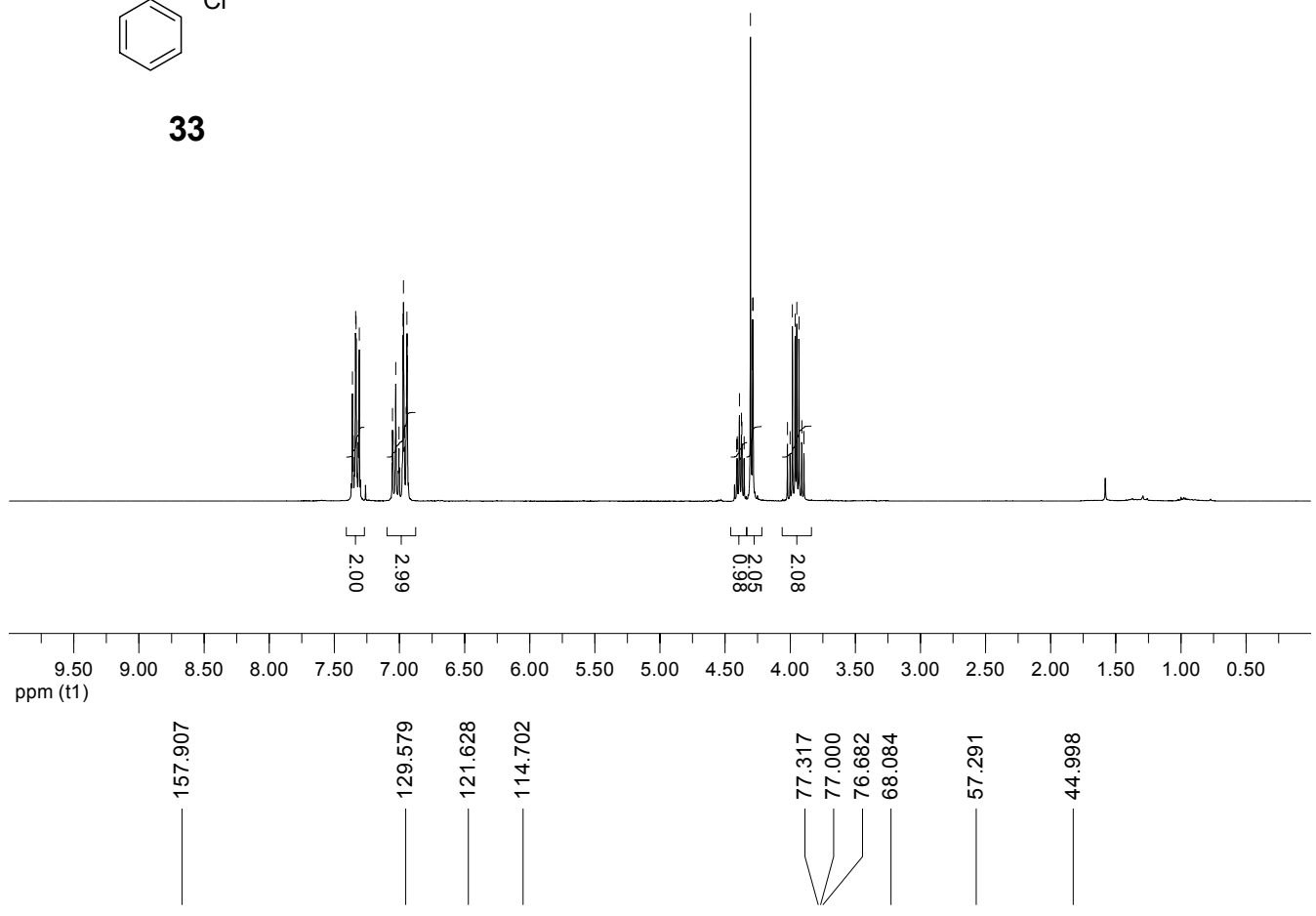

$\overbrace{C l}^{C l}$

33
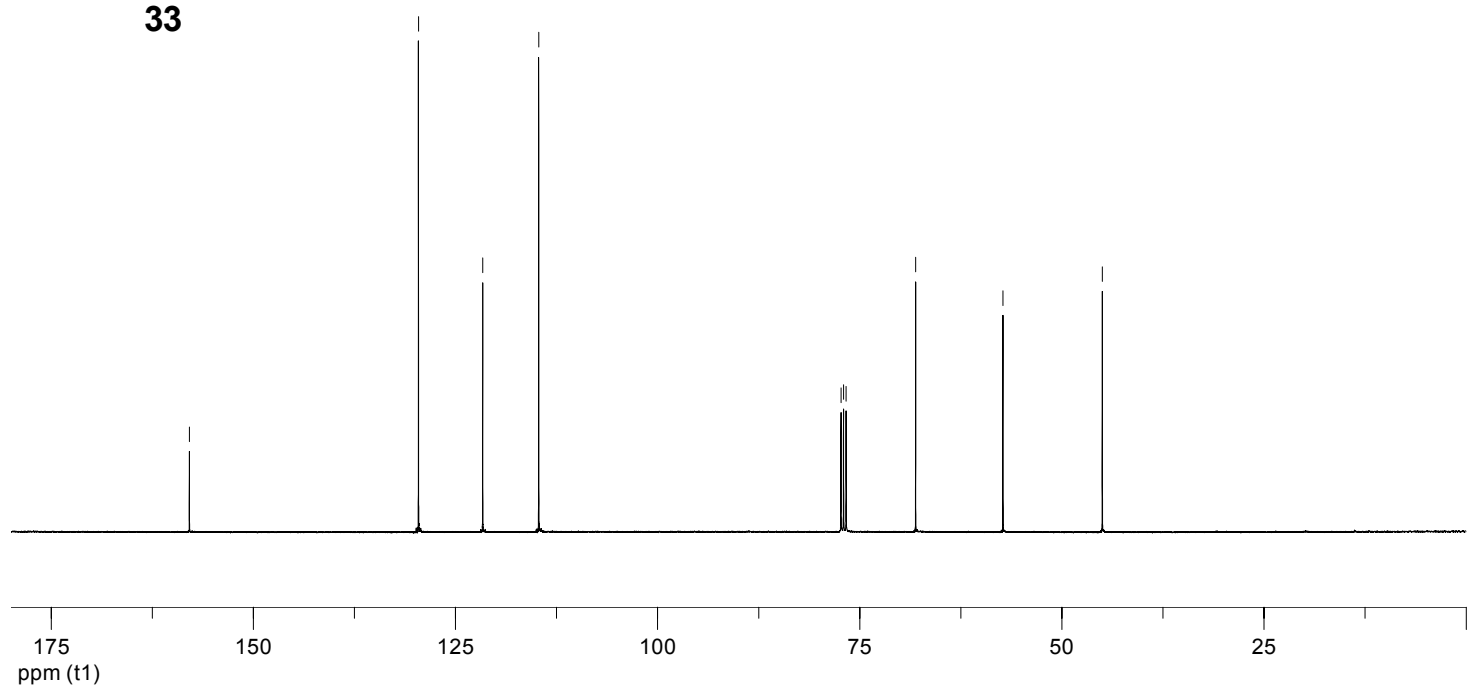


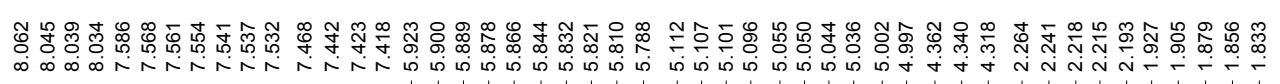

\section{$\mathrm{BzO}_{Y} \mathrm{~T}_{3} \mathrm{C}$}

35
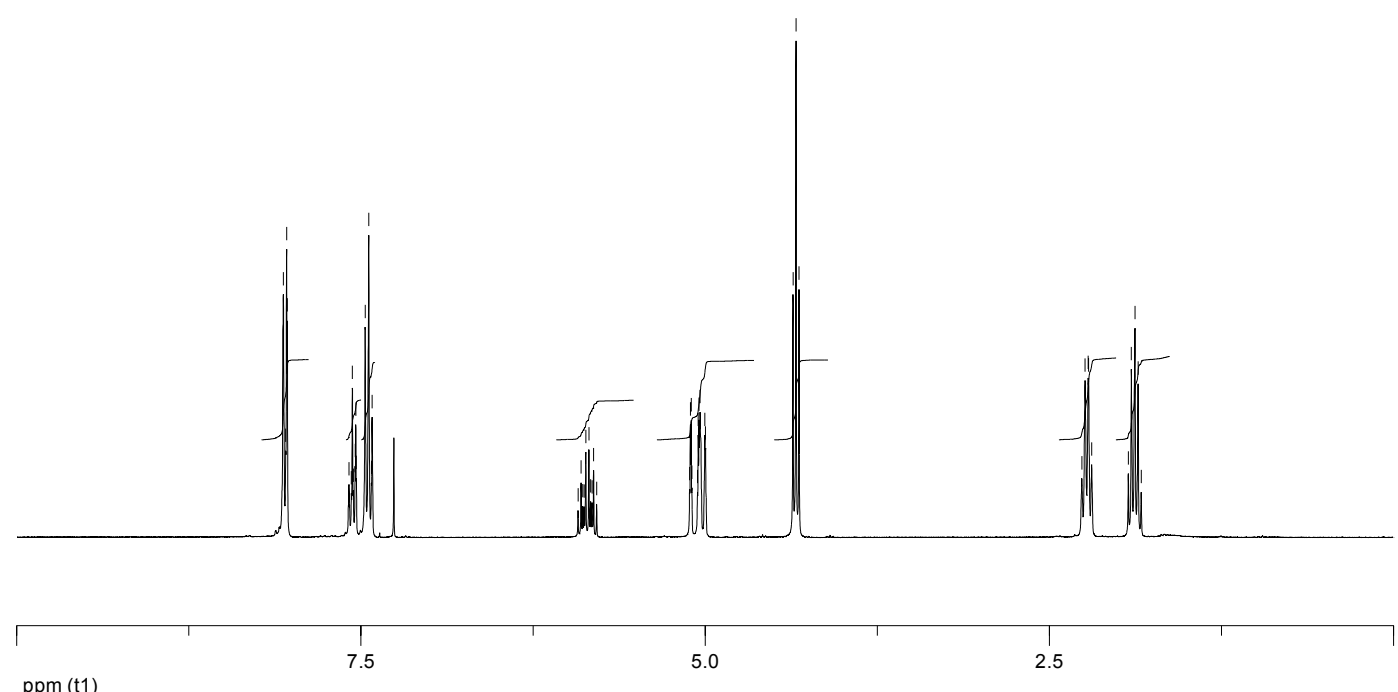

ppm (t1)<smiles>O=C(OCCCC(Cl)CCl)c1ccccc1</smiles>

36

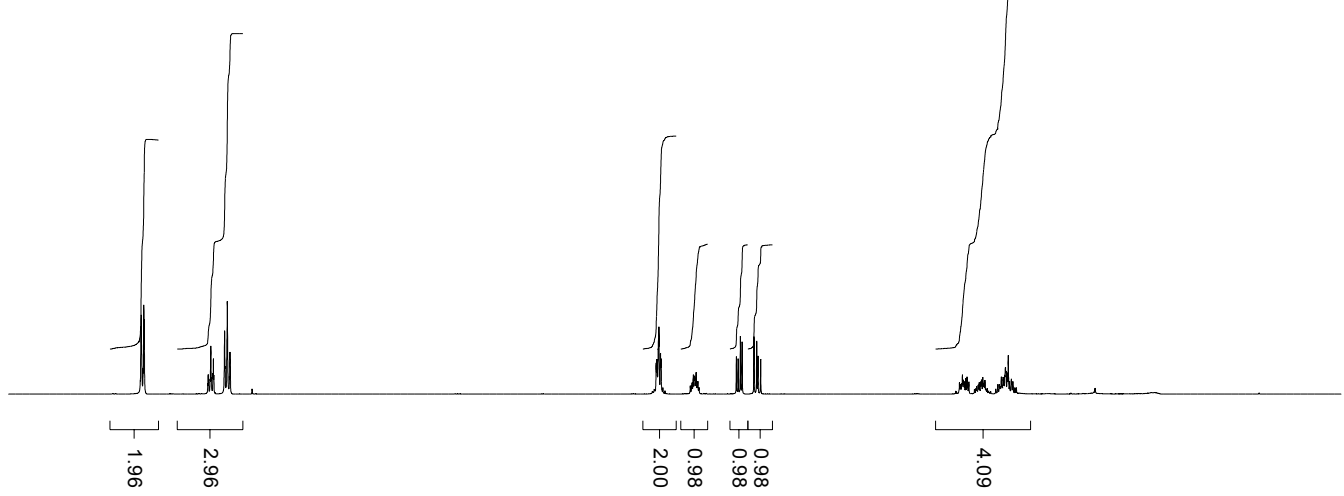

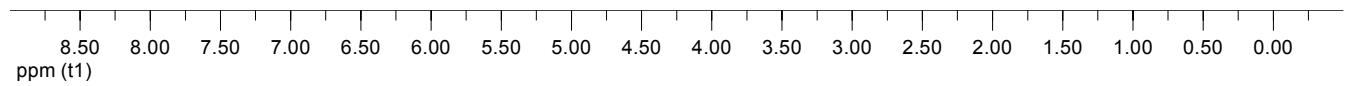



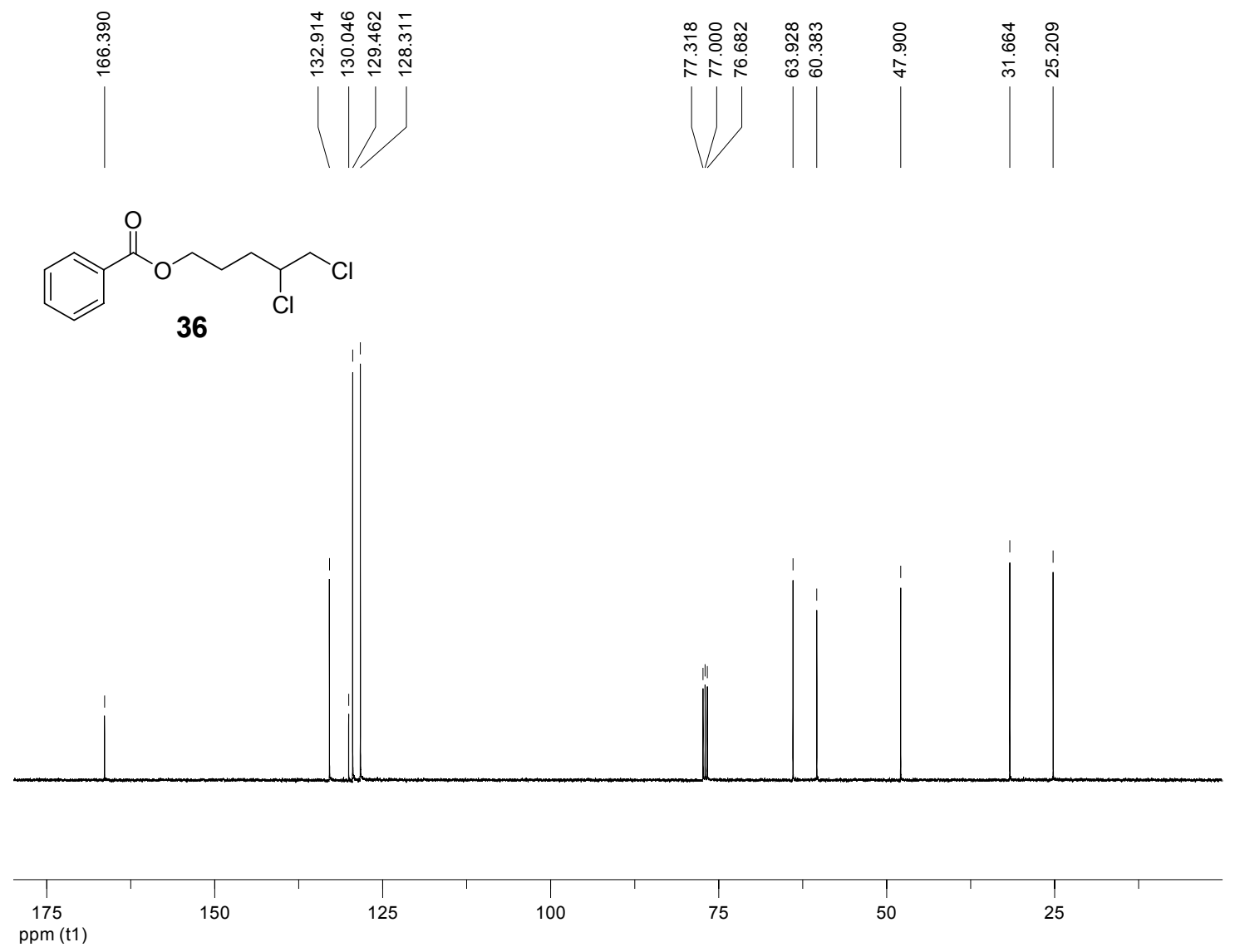

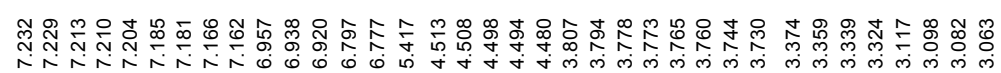
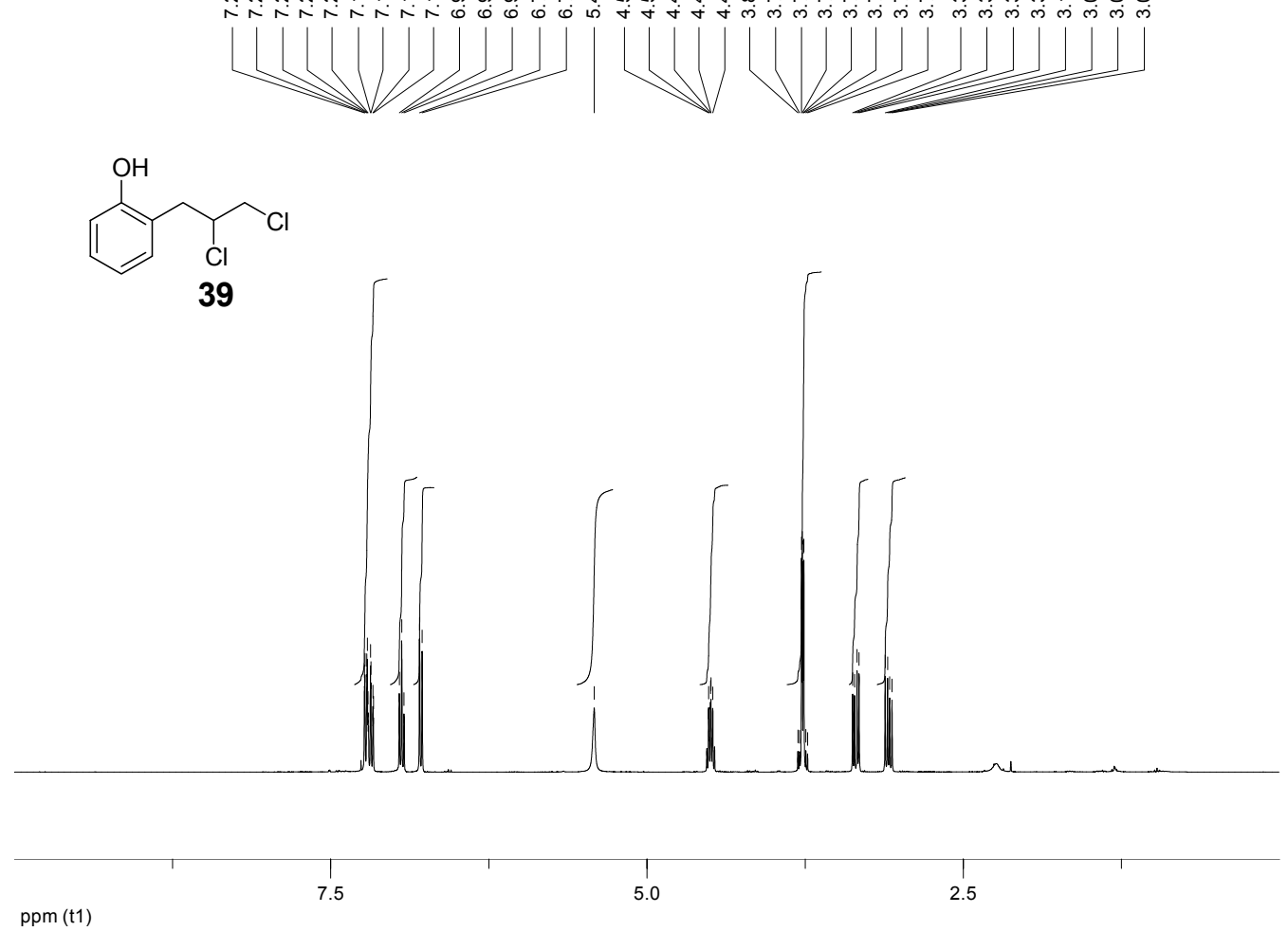


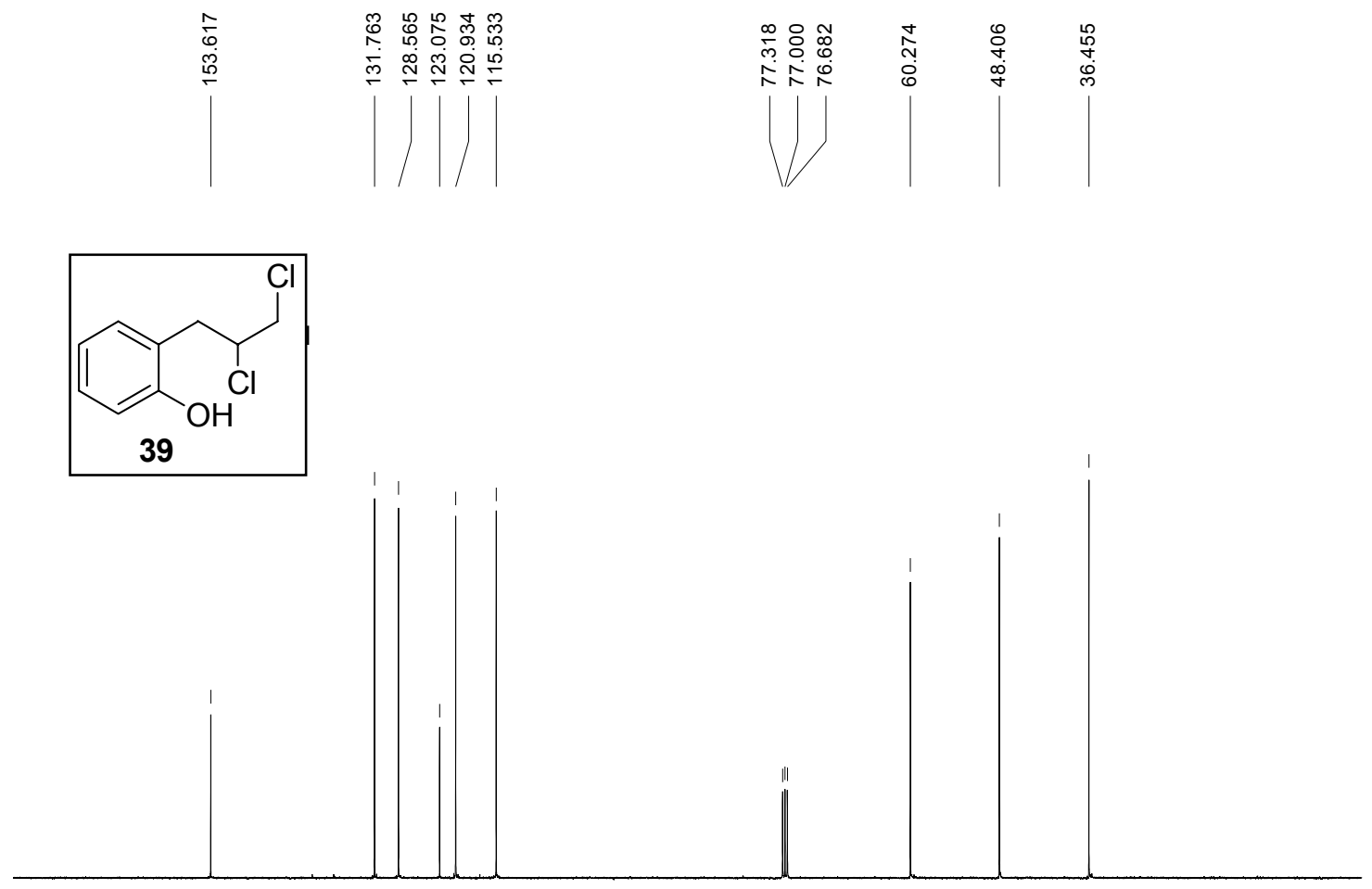

\begin{tabular}{|c|c|c|c|c|c|c|}
\hline$T$ & 1 & $\mid$ & $T$ & $T$ & $\mid$ & $\mid$ \\
\hline $\begin{array}{c}175 \\
\text { ppm (t1) }\end{array}$ & 150 & 125 & 100 & 75 & 50 & 25 \\
\hline
\end{tabular}



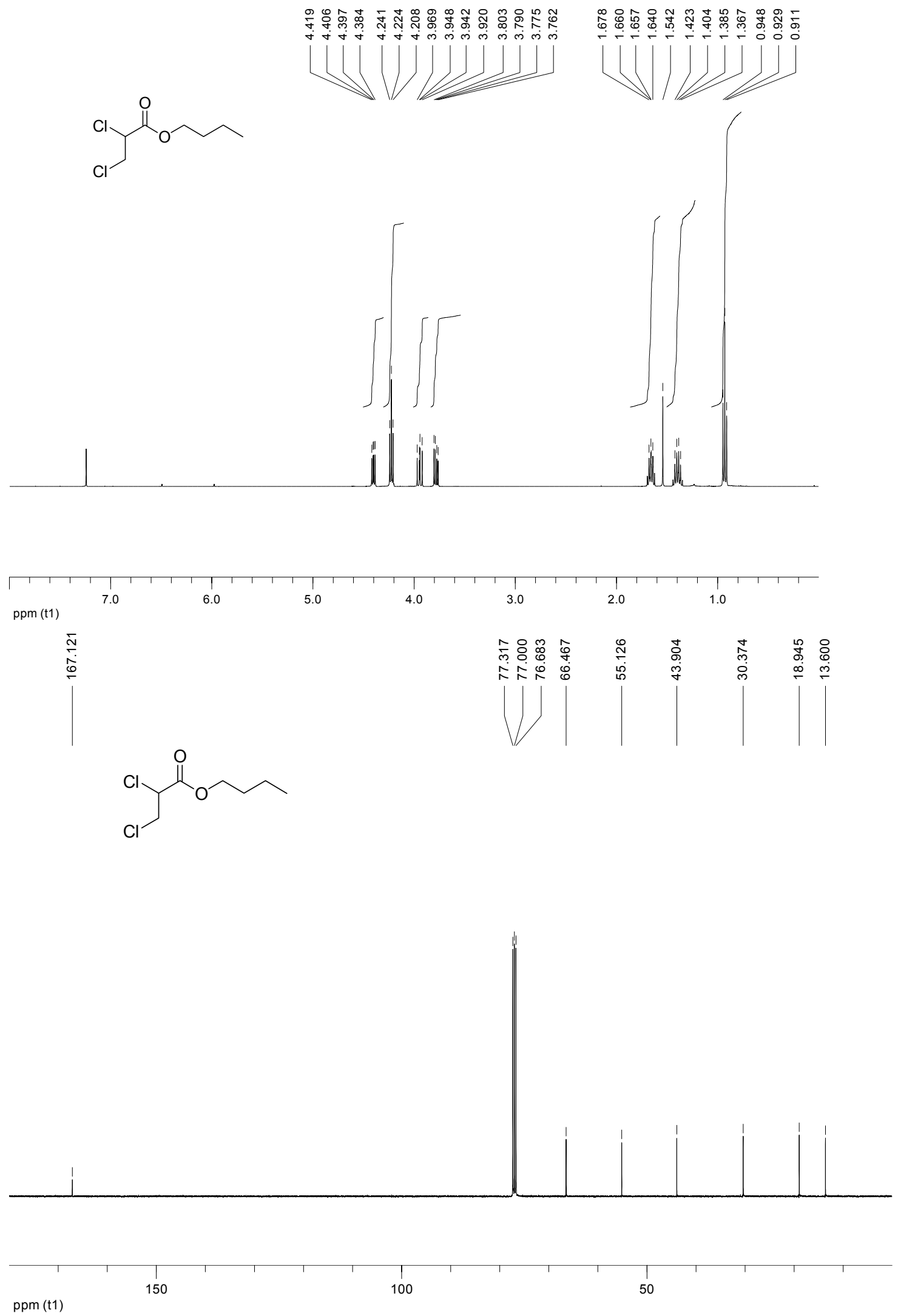

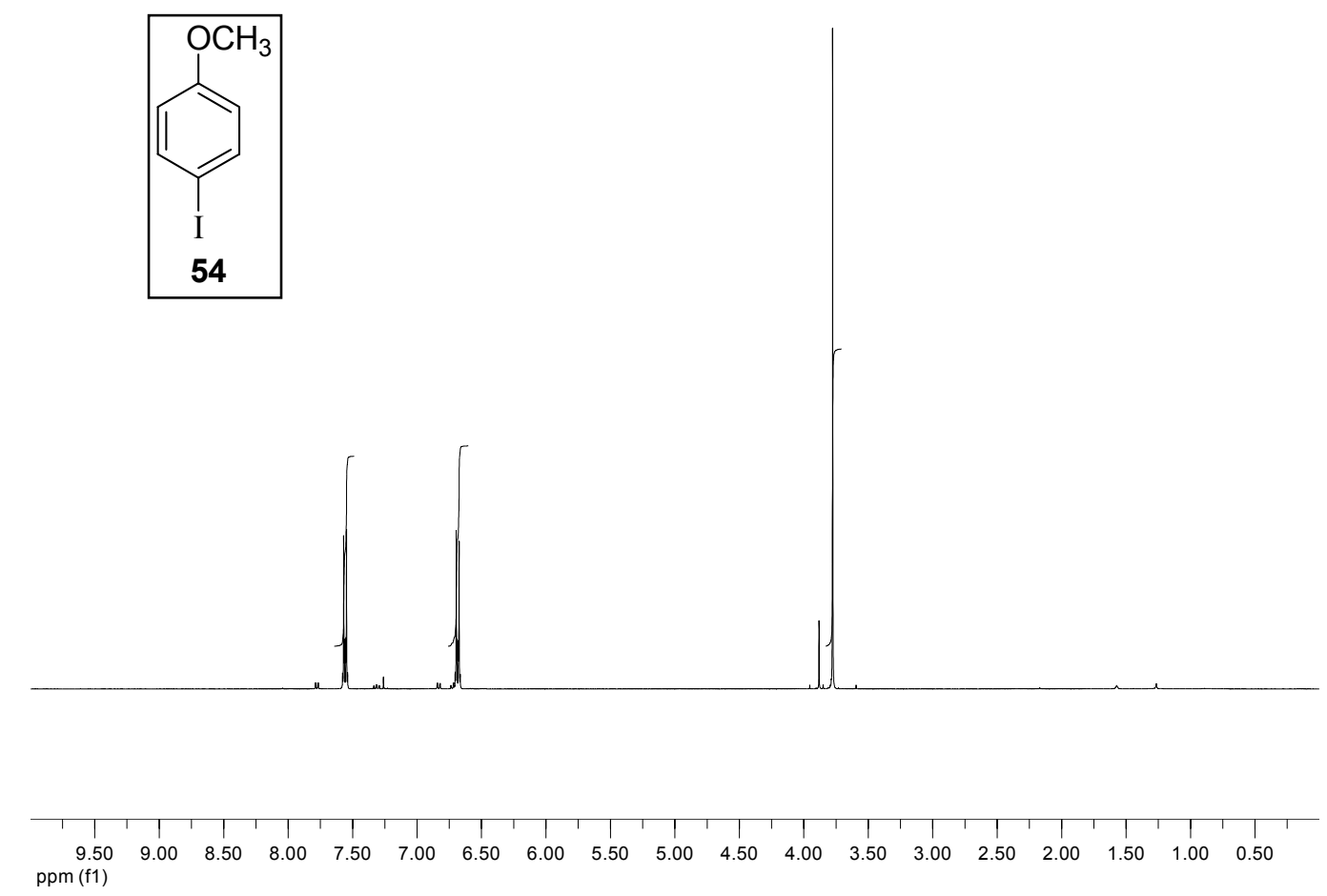

(1) Lemay, A. B.; Vulic, K. S.; Ogilvie, W. W. J. Org. Chem. 2006, 71, 3615.

(2) Smith, A. B. III; Beiger, J. J.; Davulcu, A. H.; Cox, J. M. Org. Syn. 2005, 82, 147.

(3) Hecht, S.; Frechet, J. M. J. J. Am. Chem. Soc. 1999, 121, 4084.

(4) Alternative preparation from epoxides: (a) Iranpoor, N.; Firouzabadi, H.; Azadi, R.; Ebrahimzadeh, F. Can. J. Chem. 2006, 84, 69. (b) Firouzabadi, H.; Shiriny, F. Tetrahedron, 1996, 52, 14929. (c) Iranpoor, N.; Firouzabadi, H.; Aghapour, G.; Nahid, A. Bull. Chem. Soc. Jap. 2004, 77, 1885.

(5) Kabalka, G. W. and Gooch, E. E. III. J. Org. Chem., 1980, 45, 3578.

(6) Van, T. N.; Debenedetti, S.; De Kimpe, N. Tetrahedron Lett. 2003, 44, 4199. 
(7) Horna, A.; Taborsky, J.; Churacek, J.; Dufka, O. J. Chromatography 1985, 348, 141.

(8) Kabalka, G. W.; Gooch, E. E. III. J. Org. Chem. 1980, 45, 3578.

(9) Zavada, J.; Kruipicka, J.; Kocian, O.; Pankova, M. Collection of Czechoslovak Chemical Communications 1983, 48, 3552.

(10) Bondar, D.; Liu, J.; Müller, T.; Paquette, L. A. Org. Lett. 2005, 7, 1813. 